\title{
La fondation Rockefeller et la construction d'une politique des sciences sociales en France
}

(1918-1940)

Ludovic Tournès

L'histoire des sciences de l'homme en France a considérablement étendu son territoire depuis vingt ans ${ }^{1}$. Elle a engagé des réflexions dans des domaines aussi divers que les conditions d'émergence des savoirs sur les faits sociaux ${ }^{2}$, l'évolution des disciplines (sociologie ${ }^{3}$, histoire ${ }^{4}$, anthropologie ${ }^{5} \ldots$ ), les pratiques de recherche ${ }^{6}$, les relations entre science et politique ${ }^{7}$, ou encore la collecte d'archives ${ }^{8}$. Parmi les

\footnotetext{
${ }^{1}$ Trois bilans successifs : "Une histoire des sciences de l'homme », numéro spécial de la Revue de synthèse, 3-4, 1988 ; Claude Blanckaert, Loïc Blondiaux, Laurent Loty, Marc Renneville et Nathalie Richard (eds.), L'histoire des sciences de l'homme. Trajectoires, enjeux et questions vives, Paris, L'Harmattan, 1999 ; et Jean-Michel Chapoulie, «Un cadre d'analyse pour l'histoire des sciences sociales », Revue d'histoire des sciences humaines, 2005, 13, pp. 99-126, ainsi que sa bibliographie.

2 Pierre Favre, Naissance de la science politique en France, 1870-1914, Paris, Fayard, 1989 ; "Naissance de la sciences sociale », Revue d'histoire des sciences humaines, 15, 2006.

${ }^{3}$ Outre les numéros spéciaux de la Revue française de sociologie $(20-1,1979$; 22-3, 1981 ; 26-2, 1985 ; 32-3, 1991) et des ouvrages comme celui de Laurent Mucchielli, La découverte du social. Naissance de la sociologie en France (1870-1914), Paris, La Découverte, 1998, voir le dossier récent de la Revue d'histoire des sciences humaines : "Nouveaux travaux en histoire de la sociologie » (13, 2005).

${ }^{4}$ Parmi les ouvrages récents, voir Christian Delacroix, François Dosse et Patrick Garcia, Histoire et historiens en France depuis 1945, Paris, Association pour la diffusion de la pensée française, 2003 ; André Burguière, L'école des Annales. Une histoire intellectuelle, Paris, Odile Jacob, 2006.

${ }^{5}$ On citera, entre autres: Jean Jamin, «l'histoire de l'ethnologie est-elle une histoire comme les autres ? », Revue de synthèse, 3-4, 1988, pp. 469-483; Claude Blanckaert (dir.), Les politiques de l'anthropologie. Discours et pratiques en France (1860-1940), Paris, L'Harmattan, 2001 ; «Présence de Marcel Mauss ", numéro spécial, Sociologie et sociétés, XXXVI-2, automne 2004 ; Vincent Debaene, " étudier les états de conscience. La réinvention du terrain par l'ethnologie, 1925-1939 », L'homme. Revue française d'anthropologie, 179, juillet-septembre 2006, pp. 7-62.

${ }^{6}$ "Enquête sur l'enquête". Mille neuf cent. Revue d'histoire intellectuelle, 22, 2004 ; "Pour une histoire de la recherche collective en sciences sociales", Cahiers du Centre de recherches historiques, $n^{\circ} 36,2005$.

${ }^{7}$ Emmanuelle Sibeud, Une science impériale pour l'Afrique? La construction des savoirs africanistes en France, 1878-1930, Paris, éditions de la MSH, 2002.

${ }^{8}$ Cf les programmes "Sciences sociales. Archives de la recherche", Genèses, 63, juin 2006 et http://mshdijon.u-bourgogne.fr/msh cnrs/Enquete SHS/Accueil Enquete.htm), 
chapitres encore peu explorés, celui de la dimension internationale de la construction de sciences sociales est sans doute l'un des plus complexes ${ }^{9}$. L'étude de la politique menée par les grandes fondations américaines en France constitue à cet égard un terrain de recherche extrêmement fécond, ouvert à la fin des années 1980 par le travail pionnier de Brigitte Mazon ${ }^{10}$, qui a utilisé avec profit la richesse des archives américaines pour mettre en évidence l'implication profonde des grandes fondations (Rockefeller puis Ford) dans le développement des sciences sociales en France depuis les années 1920 jusqu'au début des années 1960, à travers des projets successifs qui mènent à la création de la Maison des sciences de l'homme sous l'égide de Fernand Braudel ${ }^{11}$. Elle a ainsi montré la liaison intime qui s'opère entre les champs scientifiques national et international, et la présence d'acteurs nouveaux de la vie intellectuelle tels que les fondations philanthropiques américaines, qui jouent au même moment un rôle structurant dans le développement et l'institutionnalisation des sciences sociales aux États-Unis et dans d'autres pays, soulignée également par d'autres recherches ${ }^{12}$. Toutefois, l'étude de Mazon a laissé plusieurs problèmes non résolus et appelle de ce fait des approfondissements. D'abord parce que, centrée sur le financement de la recherche, elle n'a pas analysé les interactions intellectuelles qui s'opèrent entre les fondations et leurs interlocuteurs français, ni étudié la production scientifique réalisée à l'aide des financements américains. D'autre part parce qu'elle a négligé l'étude approfondie de l'entre-deuxguerres au profit de la période 1945-1960, considérant l'action de la fondation Rockefeller dans les années trente comme un échec ${ }^{13}$. Enfin parce que la focalisation de son étude sur l'un des acteurs du processus -la fondation Rockefeller- a entraîné de facto la sous-estimation de la dynamique propre du milieu universitaire français dans le processus d'organisation des sciences sociales avant la Deuxième guerre mondiale.

L'analyse circonstanciée de ces trois questions pemet d'affiner notre compréhension des interactions entre les champs scientifiques national et international, en montrant notamment que la contribution de la philanthropie américaine au développement des sciences sociales en France n'est pas seulement financière, mais aussi intellectuelle. La perspective retenue ici consistera non seulement à analyser la liaison entre les aspects financiers, institutionnels et intellectuels, mais aussi à considérer l'entredeux-guerres comme un moment important dans la construction d'un « champ des

audiovisuelles de la recherche (http://semioweb.msh-paris.fr/AAR/FR/); et Histoire de l'enseignement supérieur au XXe siècle (www.inrp.fr/she/picard index.htm).

9 Voir en particulier Pierre Bourdieu, "Les conditions sociales de la circulation internationale des idées ", Actes de la Recherche en Sciences Sociales, n¹45, décembre 2002, pp. 3-8; Johan Heilbron, Rémi Lenoir, Gisèle Sapiro (dir.), Pour une histoire des sciences sociales, Paris, Fayard, 2004, p. 9 ; Revue d'histoire des sciences humaines, 11, 2004, dossier sur le thème " France-EtatsUnis. Influences croisées en sciences humaines »; Jean-Michel Chapoulie, art. cité, p. 106.

${ }^{10}$ Aux origines de l'Ecole des hautes études en sciences sociales: le rôle du mécénat américain (1920-1960), Paris, Cerf, 1988.

${ }^{1}$ Sur ce processus, on consultera également, pour l'après 1945, Giuliana Gemelli, Fernand Braudel, Paris, Odile Jacob, 1995.

12 On signalera entre autres Donald Fisher, The Impact of American Foundations on the Development of British University Education 1906-1939, Ph.d., Université de Berkeley, 1977 ; Id., " The Role of Philanthropic Foundations in the Reproduction and Production of Hegemony, The Rockefeller Foundation and the Social Sciences », Sociology, 17-2, may 1983, pp. 206-233; voir aussi Bulmer Martin \& Joan, "Philanthropy and social sciences in the 1920s, Beardsley Ruml and the Laura Spelman Rockefeller Memorial, 1922-1929 », Minerva, 19, 1981, pp. 347-407; et le débat entre Bulmer et Fisher, dans Sociology, 18-4, november 1984, pp. 572-587.

${ }^{13}$ Brigitte Mazon, « La fondation Rockefeller et les sciences sociales en France, 1925-1940 », Revue française de sociologie, XXVI-2, 1985, p. 339. 
sciences sociales ${ }^{14}$ " français, qui se manifeste par l'organisation d'un programme de recherche collectif, mais aussi par la création d'un ensemble d'institutions coordonnées entre elles et unifiées par un paradigme irriguant l'ensemble des travaux qui y sont menés. Cette première institutionnalisation résulte de la conjonction de deux facteurs: un soutien financier important apporté par la fondation Rockefeller, et une vision d'ensemble des sciences sociales cristallisée au fil des négociations entreprises au début des années 1920 par les philanthropes américains avec les universitaires français. Ces négociations constituent un facteur majeur dans le processus d'organisation de la recherche en sciences sociales: pendant huit ans, entre 1924 et 1932, les représentants de la fondation Rockefeller, tenants d'une conception technicienne et utilitariste du savoir, vont discuter avec les universitaires français, en particulier les chefs de file d'une sociologie durkheimienne en crise mais désireuse de se renouveler, mais aussi avec les représentants d'autres disciplines en cours d'affirmation intellectuelle, comme l'anthropologie, l'économie ou encore les relations internationales. Dans ce processus, le rôle de la fondation ne consiste pas tant à diffuser les sciences sociales américaines qu'à promouvoir de nouvelles pratiques et de nouvelles thématiques de recherches, non seulement grâce à ses disponibilités financières, mais aussi par sa capacité à pousser les Français à coordonner leurs initiatives. C'est en ce sens qu'elle intervient comme une actrice intellectuelle, et pas seulement financière. L'analyse des négociations, qui sera menée ici dans un premier temps, permet de retracer l'entrecroisement des dynamiques des deux protagonistes et montre que l'on n'a pas tant affaire au processus de diffusion/réception d'un projet américain préalablement conçu, mais plutôt à la coproduction d'un projet franco-américain par l'ajustement progressif des objectifs des deux partenaires qui ne cessent d'évoluer au cours des négociations. La structure qui émerge de ce long processus constitue le premier stade d'institutionnalisation collective des sciences sociales: si elle est hybride dans sa forme, elle est unifiée par un paradigme commun fondé sur la recherche expérimentale, qui sera examiné dans un deuxième temps, et qui constitue le symptôme le plus évident de la naissance d'un champ français des sciences sociales au cours des années trente.

\section{LA COPRODUCTION D'UNE POLITIQUE DE RECHERCHE}

\section{Premiers contacts}

Lorsque l'envoyé du Laura Spelman Rockefeller Memorial arrive en France en février 1924, le débat relatif à l'institutionnalisation des sciences sociales y est déjà lancé depuis quelques années. De nouvelles disciplines ont en effet fleuri depuis la fin du XIXe siècle (économie, sociologie, psychologie, ethnologie..), mais restent marginales à l'université. Leur institutionnalisation franchit un cap au lendemain de la Grande guerre, notamment en raison du processus de mobilisation scientifique lancé dans la foulée de l'intervention de l'écrivain nationaliste Maurice Barrès à la Chambre

\footnotetext{
${ }^{14}$ Laurent Mucchielli, "Aux origines de la nouvelle histoire en France : l'évolution intellectuelle et la formation du champ des sciences sociales (1880-1930) », Revue de synthèse, 116-1, janvier-mars 1995, pp. 55-98.
} 
des députés en $1920^{15}$, qui affirme la nécessité d'organiser la recherche dans tous les domaines de la connaissance pour assurer le développement économique et le prestige international de la France, face à une Allemagne qui avait montré au cours de la guerre une meilleure capacité d'organisation de la science. Les trois problèmes essentiels qui se posent alors aux sciences sociales sont identiques à ceux qui affectent d'autres disciplines dans lesquelles des avancées importantes ont été réalisées depuis le début du siècle, notamment les sciences biologiques et médicales. Le premier concerne leur intégration dans l'université, qui implique une réforme de l'organisation facultaire par la création de nouveaux départements disciplinaires. Le deuxième est relatif au développement de la recherche : l'université française, conçue pour la collation des grades et non la production de connaissances $^{16}$, n'a pas prévu de structures ad hoc, et c'est pour palier cette lacune que le décret Honnorat du 31 juillet 1920 crée les instituts d'université, qui vont constituer dans l'entre-deux-guerres un vivier pour la recherche. Ce décret permet également «d'affilier à l'Université par une convention [...] des établissements privés, écoles, laboratoires, instituts, etc. complètement étrangers à l'administration et gardant après leur affiliation une autonomie propre ${ }^{17}$ ». Enfin, le troisième problème est celui du financement: si l'Etat a consenti un gros effort pour la construction de locaux depuis les années 1890, la dotation pour la recherche est notoirement insuffisante, faisant du recours aux fonds privés une constante de la politique universitaire avant 1939. Si les fondations, en particulier américaines, deviennent des partenaires du processus d'organisation de la recherche, en sciences sociales comme en sciences biologiques et médicales, c'est en raison de la conjonction de deux facteurs : la pénurie financière qui règne à l'université, et la prise de conscience de la nécessité de réorganiser son architecture intellectuelle.

C'est sur ce contexte que vient se greffer l'intervention du Laura Spelman Rockefeller Memorial (LSRM) à partir de 1924. Celui-ci est l'une des multiples organisations mises sur pied par John D. Rockefeller depuis le début du siècle. Créé en 1918, il s'oriente à partir de 1922, sous la conduite de son directeur exécutif Beardsley Ruml, vers le soutien aux sciences sociales, dans lesquelles il entend promouvoir une recherche inductive fondée sur le modèle des sciences exactes et fournissant une expertise aux décideurs politiques et aux acteurs économiques et sociaux ${ }^{18}$. Ce projet entend se déployer aux Etats-Unis, mais également en Europe, par le biais d'une stratégie de création d'institutions d'expertise dans des endroits clés dont la France, en raison de l'ancienneté de sa tradition universitaire, de son prestige intellectuel et de sa situation de grande puissance européenne, est un des principaux $^{19}$. Le premier interlocuteur que rencontrent les Américains est Charles Rist, professeur à la faculté de Droit de Paris et l'un des économistes les plus en vue de sa génération ; à la suite du débat lancé par Barrès, il a pris position en 1923 pour la création "d'instituts universitaires de science sociale qui seront chargés de

\footnotetext{
${ }^{15}$ Maurice Barrès, Pour la haute intelligence française, Paris, 1925, iii.

${ }^{16}$ Christophe Charle, La République des universitaires, Paris, Le Seuil, 1994, p. 11.

${ }^{17}$ Association des études internationales, Annuaire 1933, Paris, 1934, p. 13.

18 General Memorandum, octobre 1922 ; Principles governing the Memorial's program in the social sciences, 23 novembre 1928, Laura Spelman Rockefeller Memorial, Series III subseries 9, Box 2, Folder 10 (ci-après LSRM III-9/2/10), Rockefeller Archive Center, Pocantico Hills, États-Unis.

${ }^{19}$ Sur la nébuleuse Rockefeller et ses projets internationaux, voir Ludovic Tournès, "La fondation Rockefeller et la naissance de l'universalisme philanthropique américain, Critique Internationale, 35, avril-juin 2007, pp. 173-197.
} 
préparer l'application des méthodes scientifiques à l'action économique ${ }^{20}$ ", en formant des spécialistes appelés à travailler dans les syndicats, les chambres de commerce, les organismes gouvernementaux ou la presse. En avril $1924^{21}$, deux mois après sa rencontre à Paris avec le représentant du LSRM ${ }^{22}$, Rist est nommé advisor pour la France. De son côté, Ruml se rend à Paris en juin 1925 et demande à un universitaire américain, William Lingelbach, de réaliser une enquête sur la situation des sciences sociales ${ }^{23}$ en Europe; ce dernier rencontre à cet effet plusieurs personnalités, dont Charles Rist ${ }^{24}$, et remet à Ruml en janvier 1926 un rapport ${ }^{25}$ où il formule un jugement sévère sur les sciences sociales hexagonales, jugées trop spéculatives, et souffrant d'un manque d'organisation et de coordination en raison de la dissémination des chercheurs dans des facultés ou des instituts qui communiquent peu entre eux.

Entre-temps, dès l'été 1924, Rist a commencé son activité d'advisor. Elle consiste d'une part à sélectionner de jeunes universitaires prometteurs, auxquels la fondation accorde des bourses d'études de deux ans (fellowships ${ }^{26}$ ) pour les former à la recherche inductive dans des universités américaines et ainsi insuffler à leur retour de nouvelles méthodes dans l'université française ; Rist est invité à ce titre en mai 1925 aux Etats-Unis ${ }^{27}$ pour " prendre contact avec les institutions et les professeurs avec lesquels les fellows travailleront ${ }^{28}$ ". D'autre part, le LSRM attend de Rist qu'il formule des propositions pour l'organisation des sciences sociales ; il envoie donc à New York en décembre 1925 un rapport ${ }^{29}$, dans lequel il suggère la création d'un institut de recherches dédié à l'analyse des phénomènes économiques, non pas intégré à l'université, comme il le proposait en 1923, mais indépendant. Une telle création lui paraissant impossible dans l'immédiat, pour des raisons qu'il n'explique pas aux Américains, il propose de réunir d'abord un comité informel de personnalités scientifiques dont le rôle serait de réfléchir à l'établissement d'une série de thèmes de recherche. Enfin, il suggère le financement de structures existantes comme les Salles de travail de la faculté de Droit où les étudiants en économie se forment à l'usage des statistiques.

Ruml est déçu par ces propositions: d'abord parce que, soucieux de donner légitimité et pérennité aux sciences sociales, il veut les intégrer dans des universités et non des instituts indépendants ${ }^{30}$; d'autre part, il préfère donner une impulsion à des initiatives déjà engagées plutôt que de financer la création de nouvelles structures ; enfin, il veut développer l'ensemble des sciences sociales, alors que les

\footnotetext{
${ }^{20}$ Société des Nations. Commission de coopération intellectuelle, Enquête sur la situation du travail intellectuel. Les universités et la vie sociale en France, Par Julien Luchaire, Paris, 1923.

${ }^{21}$ Memorandum on Fellowships in the Social Sciences, 14 avril 1924, LSRM I/1/11.

${ }^{22}$ Coss à Ruml, s.d., LSRM III-6/51/535.

${ }^{23}$ Lingelbach à Ford, 5 juillet 1925, LSRM III-6/51/541.

${ }^{24}$ Lingelbach à Ruml, 21 septembre 1925, LSRM III-6/51/542.

${ }^{25}$ W. Lingelbach, Social Sciences in France, janvier 1926, LSRM III-6/51/542.

26 Ludovic Tournès, «Les élites françaises et l'américanisation: le réseau des boursiers de la fondation Rockefeller (1917-1970) », Relations internationales, n 116, novembre-décembre 2003, pp. 501-513.

${ }^{27}$ Report for the years 1923-1924, LSRM I/1/10.

${ }^{28}$ LRSM $1.2 / 50 / 380$ p. 29 , où l'on trouve la confirmation du voyage de Rist.

29 "L'organisation actuelle de l'enseignement et des recherches sociales et économiques en France et améliorations dont elles sont susceptibles. Note de M. Rist remise à la fondation Rockefeller en 1925 », Centre des archives contemporaines (ci-après CAC) 20010498, carton 111. La traduction anglaise, datée de janvier 1926, se trouve dans LSRM III-6/63/680.

30 Social Sciences. Program and Policy, 1922-1926, III-9/2/10.
} 
propositions de Rist ne concernent que l'économie ${ }^{31}$. II conclut donc qu'il est préférable d'attendre la création d'un organisme avant d'engager le LSRM dans un financement ${ }^{32}$. S'il ne rompt pas le contact avec Rist, la nomination de celui-ci comme sous-gouverneur de la Banque de France en 1926 interrompt leurs échanges ; et lorsque Rist quitte ses fonctions en mars 1929, il entame alors une carrière d'expert financier international qui l'éloignera du monde universitaire.

Avant le départ de Rist pour la Banque de France, Ruml a cherché d'autres interlocuteurs et pris contact en décembre 1925 avec l'ethnologue Marcel Mauss, qu'il invite en mai 1926 aux Etats-Unis pour visiter universités et centres de recherche, voyage au cours duquel Mauss est impressionné par l'organisation des universités américaines ${ }^{33}$. Depuis la mort de Durkheim, Mauss apparaît comme le chef de file de l'école sociologique française, relançant en 1924 l'Année sociologique dont la publication avait été interrompue par la guerre. En raison de sa position, l'organisation des sciences sociales devient un de ses thèmes de réflexion, ce dont témoignent deux textes parus en 1927, où il affirme la nécessité d'élargir les champs de recherche de la sociologie, de collaborer avec d'autres disciplines, et de développer une "organisation de nos sciences " en « ajust[ant] ensemble des ateliers de travail scientifiques ${ }^{34}$, entreprise dans laquelle il note que les Etats-Unis sont déjà engagés. Mais après le voyage de 1926, les contacts de Mauss avec le LSRM s'interrompent également, pour des raisons inconnues.

L'échec de ce premier train de négociations ne résulte pas tant d'une résistance française opposée aux projets rockefelleriens que du recul des Américains face à une situation qui ne leur semble pas favorable, et surtout d'un décalage entre les deux univers intellectuels. Le projet du LSRM est en effet difficilement soluble dans le paysage universitaire parisien, l'action à l'échelle de l'ensemble des sciences sociales envisagée par Ruml s'avérant difficile à mettre en œuvre entre des disciplines qui ont leur histoire et sont très éclatées sur le plan institutionnel. D'autre part, le fossé est grand entre la fonction d' « ingénieur social ${ }^{35}$ " que Ruml assigne au chercheur, et la figure de l'universitaire à la française dans laquelle la notion d'application du savoir tient une place marginale ; de ce point de vue, la situation des sciences sociales américaines, très ancrées dans le monde de la réforme sociale qui a été une de leur matrices, contraste avec la situation française, où plusieurs disciplines, comme la sociologie, ont déjà une légitimité universitaire, même si leur surface institutionnelle reste faible. Ceux qui auraient pu être sensible au discours philanthropique, en particulier les sociologues leplaysiens, exercent alors en dehors de l'université, qui constitue l'horizon de travail du LSRM. Enfin, celui-ci n'a pas de représentant permanent à Paris: réduits à des rencontres épisodiques lors de voyages courts, Ruml et Lingelbach peinent à saisir les ramifications universitaires françaises, qu'ils interprètent en terme d'éparpillement et de manque de coordination, à l'aune du processus de regroupement des sciences sociales en cours aux Etats-Unis, dans lequel le LSRM est impliqué au même moment.

\footnotetext{
${ }^{31}$ Ruml à Lingelbach, 27 novembre 1925, LSRM III-6/51/542.

32 Lingelbach à Ruml, 21 septembre 1925, LRSM III.6/51/542.

${ }^{33}$ Marcel Fournier, Marcel Mauss, Paris, Fayard, 1994, p. 528.

${ }^{34}$ cité dans Fournier, op. cit., p. 540.

${ }^{35}$ Memorial Policy in Social Sciences, 1922-1926, LSRM III-9/2/10.
} 


\section{La mutation du projet rockefellerien}

Si les négociations vont reprendre à partir de 1929, ce n'est pas parce que la situation française aura "mûri », mais bien parce que la fondation Rockefeller l'abordera autrement. En effet, entre 1926 et 1928, la fondation connaît une profonde réorganisation au terme de laquelle son projet et sa stratégie sortent transformés, ce qui va lui permettre de s'insérer dans le processus de construction du champ français des sciences sociales.

S'agissant du projet de la fondation, la nouvelle organisation de la philanthropie rockefellerienne qui prend effet au $1^{\text {er }}$ janvier 1929 se traduit par la fusion de divers boards, auparavant séparés, dans une nouvelle fondation Rockefeller comprenant cinq divisions : Natural Sciences, Medical Sciences, International Health, Humanities et Social Sciences, cette dernière reprenant les attributions du LSRM. Alors que dans les années 1920, l'activité de la philanthropie Rockefeller avait porté avant tout sur l'enseignement de la médecine dans les universités et l'organisation d'administrations de la santé publique, l'axe de la nouvelle fondation est « le progrès des connaissances ${ }^{36} »$. Cette réorganisation n'est pas sans liens avec les mutations du champ universitaire américain: l'apparition des sciences sociales dans l'organigramme Rockefeller en 1929 est en effet l'écho de leur éloignement croissant du monde de la réforme sociale et de leur intégration dans le champ académique, mais aussi de leur séparation avec les Humanités, un double mouvement que la Rockefeller réorganisée entérine en distinguant Social Sciences et Humanities, alors que la frontière entre les deux était encore floue au début des années vingt dans les activités du LSRM. D'autre part, alors que ce dernier raisonnait en termes d'ingénierie sociale, la Rockefeller réorganisée en départements, sur le modèle universitaire, affirme son ambition d'être désormais une organisation productrice de savoir et coordinatrice de recherches, et de renforcer ses liens avec le monde universitaire américain. La greffe de cet habitus académique sur la culture philanthropique traditionnelle, traduite par le nouvel axe directeur de la fondation, va la rendre plus sensible aux problématiques de l'université française.

Par ailleurs, les initiatives de la fondation en matière de sciences sociales sont désormais intégrées dans un projet global visant à l'élaboration d'une science totale de l'homme par la mobilisation conjointe des sciences de la nature et des sciences sociales $^{37}$. La politique parisienne de la fondation doit ainsi non seulement être comprise à l'aune de son projet mondial, mais ses actions en sciences sociales sont à bien des égards indissociables de la politique en sciences biologiques et médicales qui constitue l'autre versant de son projet intellectuel. Concrètement, la fondation envisage de créer en France une institution spécialisée dans les sciences sociales, ou, tout au moins, de soutenir un projet français ; dans cette perspective, la création du Social Science Building de l'université de Chicago, achevé en 1929 grâce au soutien financier du LSRM, lui sert manifestement de fil conducteur. D'autre part, la fondation est engagée depuis le début des années 1920 dans un projet de refonte de l'enseignement médical mondial dont le volet français se traduit par une

\footnotetext{
${ }^{36}$ Program and policy. Advancement of knowledge, 3 janvier 1929, Rockefeller Foundation (ci-après RF), Record Group 3, Series 900, Box 22, Folder 166 (ci après 3/900/22/166), Rockefeller Archive Center.

${ }^{37}$ Sur le projet rockefellerien et son évolution, voir Ludovic Tournès, "Une histoire intellectuelle des organisations internationales : le cas de la fondation Rockefeller (1913-1945) », in Emmanuel Soler, Elisabeth Lalou \& Ludovic Tournès (dir.), Les intellectuels dans la Cité de l'Antiquité à nos jours. Savoir, sociabilités, engagements, Rouen, Presses universitaires de Rouen et du Havre, à paraître en 2008.
} 
aide financière à la faculté de médecine de Strasbourg en 1925, mais aussi et surtout par le financement quasi intégral de la construction de la nouvelle faculté de médecine de Lyon, qui commence au début de l'année 1928 et est achevée deux ans plus tard. Alors que le projet lyonnais entre dans sa phase de réalisation, la fondation entame en outre au printemps 1928 des négociations avec les universitaires parisiens en vue de réaliser une opération similaire dans la capitale. Au-delà de la médecine, la Rockefeller a en point de mire une réorganisation globale de l'enseignement supérieur français, dont les grandes lignes sont esquissées en octobre 1928 à New York lors d'une réunion au sommet destinée à préciser la nouvelle stratégie de la fondation alors que la réorganisation en divisions entre dans sa dernière longueur; la fondation envisage de substituer aux actions isolées engagées par les différents boards dans les années vingt (avec la Faculté des sciences, la Faculté de médecine, mais aussi de nombreux instituts de recherche) une action à l'échelle de l'ensemble de l'université de Paris ${ }^{38}$, dont les Américains connaissent bien la pauvreté chronique ${ }^{39}$. A partir de 1929 , les contacts avec les Français s'inscriront donc dans un plan implicite de refonte globale de l'université de Paris, dont la pièce maîtresse est la construction d'une nouvelle faculté de médecine. Même si ce projet n'aboutira pas (la fondation retire son offre de financement à la fin de l'année 1932), ce projet sert de sous-bassement à la première construction institutionnelle des science sociales en France.

Enfin, c'est aussi la stratégie de la fondation sur le terrain qui évolue avec la réorganisation. D'abord parce que le personnel change: en 1929-1930, la Social Sciences Division (SSD) a recruté deux officers fins connaisseurs de l'Europe, contrairement à leurs prédécesseurs du LSRM : John Van Sickle, qui travaillé à l'ambassade américaine à Paris en 1919-1920, avant d'exercer les fonctions de conseiller technique du gouvernement autrichien jusqu'en 1923, travail qui a fourni la matière à sa thèse d'économie ; et Tracy Kittredge, ex-coordinateur pour le Nord de la France de la Commission for the Relief in Belgium créée en 1914 par Herbert Hoover, puis Secrétaire général de la Fédération Internationale des Sociétés de la Croix Rouge et du Croissant Rouge. Les deux hommes seront les interlocuteurs permanents des universitaires français jusqu'en 1940. Par ailleurs, aux contacts épisodiques menés par Ruml au début des années 1920 vont se substituer désormais des négociations au plus haut niveau administratif. Lors de la réorganisation, la fondation a en effet créé un poste de Vice-président chargé spécifiquement de la politique européenne, poste attribué à Selskar Gunn. Ce médecin, qui coordonne depuis 1917 les activités européennes de la fondation en matière de santé publique et d'enseignement médical, a acquis une bonne connaissance du complexe politico-administratif hexagonal, fruit de plusieurs années de négociations avec l'administration et les gouvernements dans le cadre des projets successifs de la fondation en France, que ce soit la campagne antituberculeuse de 1917-1922, le soutien à l'Office national d'hygiène sociale du ministère de l'Hygiène à partir de 1925, ou encore les actions en matière d'enseignement médical mentionnées plus haut. Gunn va ainsi mettre à la disposition des officers de la SSD le réseau de contacts construit par les autres boards de la philanthropie Rockefeller avant la réorganisation, un appui logistique qui avait fait défaut aux représentants du

\footnotetext{
${ }^{38}$ Agenda For Office Conference, 20 novembre 1928, RF 1.1/500A/5/55.

${ }^{39}$ Sessions de la chambre des députés, 23 novembre 1928 et du Sénat, 24 et 26 décembre 1928, citées dans un memorandum du 7 février 1929, RF 1.1/500A/5/55. Voir notamment la deuxième séance du 23 novembre 1928, Journal Officiel de la République française. Débats parlementaires. Assemblée nationale, novembre 1928, p. 2828.
} 
LSRM quelques années plus tôt. Cette nouvelle orientation est fondamentale pour comprendre la reprise des négociations et la forme qu'elles prennent.

\section{Négociations simultanées}

A partir de ce moment en effet, la Rockefeller établit des contacts de plus en plus étroits avec une partie des représentants des sciences sociales français, se trouvant intégrée dans les problématiques intellectuelles, mais aussi les rapports de forces personnels et institutionnels du milieu universitaire parisien. Cette deuxième phase de négociations qui s'ouvre en 1929 ne se réduit pas à un face à face francoaméricain, mais comporte plusieurs négociations simultanées entre la Rockefeller et des acteurs qui ont des positions divergentes : Sébastien Charléty, Marcel Mauss et Charles Rist.

Les premiers contacts de la fondation avec Charléty remontent à 1920 : celui-ci était alors Directeur général de l'instruction publique et des beaux-arts pour l'AlsaceLorraine $^{40}$ et avait reçu les Américains qui étudiaient la possibilité d'un soutien financier à la faculté de médecine de l'université de Strasbourg ${ }^{41}$. Au début de 1928, alors qu'il a été promu Recteur de l'université de Paris l'année précédente ${ }^{42}$, les Américains le sollicitent à nouveau pour évoquer leur projet de financer la construction d'une nouvelle faculté de médecine à Paris ${ }^{43}$; les discussions s'intensifient au printemps 1930, après que la fondation a annoncé en décembre 1929 qu'elle était prête à investir 12 millions de dollars ${ }^{44}$ dans l'opération. Entretemps, la Social Sciences Division a envoyé de son côté en mai-juin 1929 à Paris le politologue de l'université de Chicago Charles Merriam ${ }^{45}$, pour réouvrir les discussions interrompues depuis 1926. Mais c'est seulement au printemps 1930 qu'elles démarrent vraiment, grâce à la dynamique lancée par le projet de faculté de médecine : Selskar Gunn, qui rencontre régulièrement Charléty depuis 1928, aborde pour la première fois avec lui la question des sciences sociales le 21 mai $1930^{46}$. Désormais, à chacune de leurs entrevues, les deux hommes évoqueront à la fois les problèmes relatifs à la faculté de médecine et ceux qui concernent les sciences sociales.

Lors de sa visite parisienne, Charles Merriam n'a pas rencontré Rist, alors en mission en Roumanie, mais a repris contact avec Mauss. Celui-ci constitue a priori l'interlocuteur idéal en raison de la nouvelle orientation du projet rockefellerien : il est en effet, en tant que directeur de l'Institut d'ethnologie, rattaché institutionnellement au Muséum d'histoire naturelle et peut de ce fait constituer une interface entre sciences de la nature et sciences sociales dont la mise en synergie est maintenant au cœur de l'agenda scientifique de la fondation. Si en 1925, l'économie, la sociologie et la science politique constituaient l'horizon essentiel du LSRM, la Social

\footnotetext{
${ }^{40}$ Dossier de Sébastien Charléty, Archives Nationales (ci-après AN) AJ16 5920.

41 Annual report of the medical division of the Commission for the Prevention of Tuberculosis in France, 1920, RF 1.1/500T/30/271.

42 II occupe ce poste jusqu'à sa retraite en 1937 (AN AJ16/5920).

${ }^{43}$ Richard M. Pearce Diary, 17 janvier 1928, RF 1.1/500A/5/55.

44 IHB Minutes, 13 novembre 1929, RF 1.1/500A/5/53.

45 Pierre-Yves Saunier, "Paris in the Springtime : un voyage de science sociales en 1929 », Revue d'histoire des sciences humaines, 2004, 11, pp. 127-156.

${ }^{46}$ Selskar M. Gunn (ci-après SMG) diary, 21 mai 1930, RF 12.1 ; voir aussi SMG, Interview with S. Charléty, 29 septembre 1930, RF 1.1/500A/6/56
} 
Sciences Division s'intéresse désormais également à d'autres disciplines plus proches des sciences de la nature, telles que la psychologie et l'anthropologie. Ce sont sans doute ces questions que Merriam aborde au cours de son entrevue avec Mauss, qu'il conclue en lui demandant de rédiger un projet de demande de subvention. Six mois plus tard, en décembre 1929, Mauss envoie trois projets à la fondation, dont il adresse copie à Sébastien Charléty. Le premier porte sur l'anthropologie: Mauss suggère des subventions destinées à développer la recherche, et à permettre le regroupement de « tous les établissements savants (de l'État) s'occupant de l'Homme, de ses sociétés dites inférieures ou exotiques, et de sa préhistoire ${ }^{47} \%$. Le deuxième projet propose une subvention à l'institut de psychologie de l'université de Paris ${ }^{48}$. Le troisième, le plus ambitieux, envisage de "regrouper et coordonner» toutes les structures existantes dans un « vaste immeuble dédié aux sciences humaines et sociales ${ }^{49}$ " dont le coût est estimé par Mauss à 25 millions de francs, soit 2 millions de dollars ; informé du projet de faculté de médecine, l'ethnologue sait que cette somme est largement à la portée du portefeuille rockefellerien. L'institut serait rattaché à une sixième section de l'École pratique des hautes études (EPHE) créée à cet effet et consacrée « aux recherches économiques et sociales ${ }^{50}$ "; cette solution consacrerait l'intégration de la totalité des sciences sociales dans l'université, Mauss ne cachant pas sa réticence vis-à-vis des instituts privés.

Parallèlement, les officers ont repris les discussions avec Rist rentré d'Europe centrale, et en mars 1930, John Van Sickle sollicite son avis sur le projet de Mauss : Rist critique la faible part qu'y tient l'économie et juge irréaliste le regroupement des sciences sociales en raison des affrontements personnels et scientifiques qu'une telle formule risque de provoquer. II considère qu'il faut « commencer modestement par un institut de recherche en économie ${ }^{51}$ » qui, insiste-t-il, doit être indépendant de l'université. Au cours du printemps 1930, Rist réitère à plusieurs reprises ces critiques $^{52}$, et les officers, qui trouvent également le projet de Mauss confus ${ }^{53}$, font savoir à celui-ci au mois de mai qu'il ne leur paraît pas viable. Mais l'épisode n'est pas sans entrainer des tensions entre Rist et les Américains : l'économiste goûte modérément les discussions parallèles entamées par les officers et redoute manifestement de voir se constituer un axe Charléty-Mauss dont la volonté d'agir à l'échelle de l'ensemble des sciences sociales dans le cadre universitaire est bien plus proche de la stratégie de la fondation que ses propres propositions relatives à la création d'un institut indépendant. II envisage alors de démissionner de ses fonctions

\footnotetext{
${ }^{47}$ Marcel Mauss, Projet concernant les sciences anthropologiques et ethnologiques, décembre 1929, CAC 20010498/111.

${ }^{48}$ Id, Projet de subvention à l'institut de psychologie de l'université de Paris.

${ }^{49} \mathrm{Id}$, Projet d'un institut de recherche des sciences sociales à l'université de Paris.

${ }^{50}$ Id, Note générale.

51 John Van Sickle (ci-après JVS) Diary, 12 mars 1930, RF 12.1.

52 JVS Diary, 30 avril et 20 mai 1930, RF 12.1; SMG diary, 20 mai 1930, RF 12.1

${ }^{53}$ SMG diary, 16 mai 1930, RF 12.1. Le projet de Mauss, sans doute rédigé rapidement, est parsemé de fautes de frappe et ne brille pas par sa clarté, notamment dans sa description des "sciences sociales et humaines ", divisées en quatre catégories : "1/ l'anthropologie sous toutes ses formes: somatologie, races, etc.. préhistoire, etc... biométrique, etc.. 2/ la psychologie humaine et comparée, normale et pathologique, pure et appliquée... 3/ sciences sociales pures (générales, comparées), sociétés animales) [sic], rapports avec l'anthropologie ; sciences comparées des religions, du droit, de l'économie, de la technique, de l'esthétique, de la linguistique, de la statistique, de la géographie humaine. 4/ sciences sociales appliquées : politique, droit international, etc... " (Note générale, op. cit.).
} 
de d'advisor ${ }^{54}$, avant qu'une conciliation n'intervienne au cours d'un dîner à son domicile à l'automne $1930^{55}$. Les Américains, qui souhaitent garder le contact avec lui, s'attacheront désormais à éviter tout risque de conflit. A la fin de l'année 1930, ils sont cependant conscients qu'il sera difficile de concilier le projet de Charléty et celui de Rist ${ }^{56}$. Mais ce dernier, pensant être assuré désormais du soutien de la fondation, pousse son avantage en envoyant en décembre à Charléty une copie de son rapport de 1925, dans lequel la seule modification par rapport à la version originale réside dans l'affirmation que, désormais, les conditions sont réunies pour la création d'un institut ${ }^{57}$.

Si les négociations piétinent au cours de l'année 1930, c'est donc essentiellement en raison de l'affrontement entre Rist et Mauss, Charléty observant de son côté une prudente neutralité. C'est au début de 1931 que la situation se débloque avec l'entrée en lice d'un nouvel interlocuteur dans le processus : le sociologue Célestin Bouglé. En effet, en janvier 1931, Charléty, conscient que les conflits franco-français risquent de compromettre le financement américain, convoque Rist et Mauss dans son bureau afin de débloquer les discussions qui n'ont pas avancé depuis le printemps $1930^{58}$, et invite également Bouglé. Le détail de la discusion n'est pas connu, mais le résultat en est clair: la cohabitation des deux projets (celui d'un institut indépendant défendu par Rist et celui d'une organisation fédérant les sciences sociales défendue par Mauss et Charléty), qui semblait impossible quelques mois plus tôt, est désormais entérinée. Un tel déblocage n'a pu être obtenu que par l'éviction de Mauss du processus au profit de Bouglé, et ce, manifestement sous la pression du recteur. Elle est en partie liée à des raisons personnelles : à l'évidence, Bouglé, qui connaît Rist depuis la fin du XIXe siècle, entretient de bons rapports avec lui, alors que les relations de l'économiste et de l'ethnologues sont exécrables. Mais il y a d'autres raisons : si Mauss a l'envergure intellectuelle requise pour élaborer un projet de regroupement des sciences sociales, les Américains n'ont guère confiance dans ses qualités d'organisateur, et sa position marginale par rapport à l'université (il est professeur à l'Institut d'ethnologie jusqu'à son élection au Collège de France en novembre 1930) le dessert ${ }^{59}$. Bouglé, lui, occupe une place centrale dans l'institution (il est titulaire de la chaire d'histoire de l'économie sociale de la Sorbonne depuis $1919^{60}$ ) et ses qualités d'organisateur (il est directeur adjoint de l'École normale supérieure), son entregent institutionnel (il est membre de multiples commissions) et sa légitimité intellectuelle (il est l'auteur de nombreux ouvrages) le rendent apte à concevoir un projet que Charléty portera sur le plan administratif. Ce dernier, en marginalisant Mauss, a ménagé la susceptibilité de Rist tout en conservant ce qui lui tient à cœur depuis le début : une organisation globale des sciences sociales.

\footnotetext{
${ }^{54}$ SMG diary, 27 mai, 12 juin, 23 juin 1930, RF 12.1.

55 JVS Diary, 29 septembre 1930, RF 12.1.

56 JVS Diary, 9 décembre 1930, RF 12.1.

${ }^{5}$ Rist à Charléty, 20 décembre 1930, CAC 20010498/111.

58 JVS Diary, 16 janvier 1931, RF 12.1.

59 Le positionnement politique de Mauss, mentionné par Mazon (op. cit., pp. 48-49) nous semble secondaire dans son éviction.

${ }^{60}$ Dossier de Célestin Bouglé, AN AJ16/5885.
} 


\section{Célestin Bouglé et la coordination de la recherche}

Au cours des rencontres avec les Américains qui suivent cette réunion, les officers acceptent la cohabitation des deux projets puisqu'il semble impossible de trancher en faveur de Charléty sans provoquer le retrait de Rist. Ils demandent alors aux deux parties de formaliser leurs propositions ${ }^{61}$. C'est lors de cette phase de formalisation que Bouglé s'impose comme l'interlocuteur privilégié des Américains. S'il peut jouer ce rôle, c'est non seulement en raison de son entregent et de sa multipositionnalité, mais aussi parce qu'il est l'un des principaux dépositaires de l'héritage durkheimien, dont l'ambition de réaliser une synthèse des sciences sociales préexiste à l'arrivée de la philanthropie américaine, et dont l'évolution au cours des années 1920 l'amène à rentrer en adéquation avec les projets de la Rockefeller. En effet, décimée par la Grande guerre, l'école durkheimienne est alors fragilisée, à un moment où ses positions institutionnelles restent faibles, et alors que son territoire intellectuel est mal balisé, puisque le durkheimisme se caractérise avant tout par un point de vue sur les sciences sociales plus que par des thématiques d'études propres. D'autre part, sa prétention à occuper une situation surplombante par rapport aux autres disciplines, formulée dès la fin du XIXe siècle, se voit contestée à la fin des années 1920 par l'émergence de l'école historique des Annales, dont le projet intellectuel empiète sur ses terres en affirmant son ambition de favoriser l'interdisciplinarité et de faire dialoguer les historiens avec les chercheurs travaillant sur les «sociétés et les économies contemporaines ${ }^{62}$ ", alors que, depuis l'interruption de la deuxième série des Annales sociologiques à la fin de 1927, les durkheimiens n'ont plus d'organe officiel. Conscient de la crise intellectuelle de sa discipline, Marcel Mauss note alors qu'une des voies du renouveau consiste à abandonner la dimension spéculative, " systématique et généralisatrice " pour se consacrer à des recherches " descripti[ves] $]^{63}$ " sur des thématiques précises, afin de se constituer un territoire intellectuel clairement identifié face à d'autres sciences sociales.

L'ambition de la sociologie reste donc intacte, mais prend un nouveau visage dans les années trente: il ne s'agira plus tant pour elle de diffuser l'esprit sociologique dans les autres disciplines que de fédérer des recherches de terrain qu'elle souhaite voir se développer, en sociologie et ailleurs. L'émergence de l'idée de " coordination » des sciences sociales est le principal symptôme de cette évolution, et c'est Bouglé qui en sera le plus ardent propagandiste. L'apparition de cette notion dans la pensée de Bouglé remonte au colloque organisé à Chicago en décembre 1929 pour l'inauguration du Social Science Building, auquel le sociologue a été convié par Charles Merriam lors de sa visite à Paris ${ }^{64}$. Bouglé y prononce une intervention consacrée aux relations entre la sociologie et les autres disciplines qui apparaît en décalage par rapport aux textes de ses collègues américains, qui ne traitent que de coordination, de programmes de recherches, d'enquêtes collectives et d'interdisciplinarité. Tout juste le Français fait-il allusion au rôle de la sociologie dans la mise en œuvre de recherches «objectives, à la fois comparatives et synthétiques $^{65} »$. Mais c'est au cours de ce séjour qu'il prend conscience de la réalité intellectuelle et physique (l'existence d'un bâtiment unique) de la coordination,

\footnotetext{
61 JVS Diary, 20 avril 1931, RF 12.1.

${ }^{62}$ Annales d'histoire économique et sociale, $\mathrm{n}^{\circ} 1$, janvier 1929, p. 1.

${ }^{63}$ Marcel Mauss, « La sociologie en France depuis 1914 », in : La science française, nouvelle édition entièrement refondue, tome I, Paris, 1934, p. 43.

${ }^{64}$ Rapport du CDS pour 1929, AN 61AJ/97.

${ }^{65}$ Célestin Bouglé, «The present Tendency of the Social Sciences in France », in Leonard D. White (ed), The new Social Science, Chicago, 1930, p. 66.
} 
comme on peut le voir dans le compte rendu des actes du colloque (parus en 1930) qu'il rédige en 1934 pour le premier numéro des Annales Sociologiques ${ }^{66}$ : il y note que l'institut de Chicago a pour but de "promouvoir les recherches positives en matière de sciences sociales » et de "coordonner en facilitant le rapprochement et la collaboration des diverses disciplines ${ }^{67}$ ". Mais surtout, il propose une réinterprétation de sa propre contribution de 1929, écrivant ainsi que «le délégué français, dans une communication sur les tendances actuelles des sciences sociales en France, indiqua comment, surtout grâce à l'influence de Durkheim, on avait cherché à résoudre en France ce problème qui préoccupait nos collègues américains: orienter vers des synthèses positives les diverses disciplines s'appliquant aux choses sociales ${ }^{68}$ ». Le glissement sémantique est à peine perceptible, mais hautement révélateur: les recherches «comparatives et synthétiques " évoquées dans sa communication de 1929, sont devenues dans le compte rendu de 1934 des synthèses "positives ", c'est-à-dire, dans l'esprit de Bouglé, issues de recherches de terrain interdisciplinaires sous la coordination intellectuelle de la sociologie. A partir du début des années trente, la notion de " coordination " des sciences sociales devient une constante de la pensée de Bouglé $^{69}$ et va le mettre en phase avec les projets de la fondation.

On est alors au printemps 1931, et les officers dressent un bilan négatif des fellowships : sur les dix neuf fellows sélectionnés par Rist entre 1924 et $1931^{70}$, deux seulement ont entamé à cette date une carrière académique en France. La faculté de droit ne s'étant pas révélé un bon vivier de fellows, les officers s'intéressent désormais à l'Ecole normale supérieure, dont ils commencent à comprennent la place centrale dans l'université française, donnée qui avait échappé à Ruml et Lingelbach en 1924. Bouglé devient alors incontournable : en juin 1931, Van Sickle le rencontre deux fois et vient assister aux examens d'entrée de l'ENS, puis prend contact, au ministère de l'Instruction Publique, avec le directeur de l'Enseignement supérieur Jacques Cavalier pour organiser l'octroi des fellowships à des normaliens, de façon à ce que leur séjour à l'étranger intervienne au bon moment dans leur cursus. A la fin de 1931, Van Sickle et Bouglé établissent une première liste de candidats possibles ${ }^{71}$, tandis que les Américains demandent à Charléty des renseignements sur les instituts d'université afin de préparer l'octroi d'une subvention pour certains d'entre eux ${ }^{72}$. Avec le matériau collecté par les services du recteur, Tracy Kittredge rédige un long memorandum achevé au mois de juin 1932. Synthétisant les propositions respectives des officers américains et des universitaires français depuis 1925, Kittredge formalise pour la première fois le projet Rockefeller,

\footnotetext{
${ }^{66}$ Celles-ci constituent en fait la troisième série de L'Année sociologique.

${ }^{67}$ Annales sociologiques, Série A, fascicule 1, 1934, p. 93.

${ }^{68} \mathrm{Ibid}, \mathrm{p} .96$.

${ }^{69}$ Bilan de la sociologie française contemporaine, Paris, Alcan, 1935, p. vi et 169 ; « L'organisation de la recherche sociale en France ", Annales de l'université de Paris, 1936, pp. 225-233; Centre d'étude de politique étrangère, Les sciences sociales en France. Enseignement et recherche, Paris, 1937, pp. 45 sq.

70 André Philip, Ernest Teilhac, Marthe Bossavy, Jean-Marie Claoué, J.L. Gachon, Madeleine Leliepvre, Pierre Denoyer, Paul Devinat, Désiré Pasquet (LRSM Dockets 1924-1925, 1/1/9, 1/1/11, 1/2/14), Vsevolod Basanoff, Edouard Lavergne, Pierre Mariotte, Pierre Winckler, Jean Legrand, Raymond Sachot, Robert Valeur, Jacques Lambert, François Trévoux, Paul Baratier. Voir Ludovic Tournès, "Les boursiers français du programme Fellowships and Scholarships de la Fondation Rockefeller, 1917-1970 », base de données, site de l'Institut Pasteur, http://www.pasteur.fr/infosci/archives/f-rock.html, [En ligne], 2003.

71 JVS Diary, 11 décembre 1931, RF 12.1.

${ }^{72}$ Charléty aux directeurs d'instituts, 15 décembre 1931, CAC 20010498/111.
} 
qui apparaît bien plus comme le résultat de ce processus de négociations que comme celui d'une élaboration interne à la fondation. Kittredge envisage plusieurs possibilités pour faire de Paris un centre majeur des sciences sociales :

$1 /$ la création d'une faculté, projet qu'il juge trop ambitieux pour être réalisable à court terme ;

2/ la création d'un institut d'université, formule plus modeste qui éviterait une réorganisation globale du système universitaire, et pourrait servir de base à un " programme coordonné de recherches en sciences sociales ${ }^{73}$ ".

3/la création d'une section des sciences sociales à l'EPHE, comme le suggérait Mauss.

C'est à la suite de ce rapport que la fondation accorde au printemps 1932 ses premiers financements à quelques instituts ${ }^{74}$ : L'Institut de droit comparé, l'Institut d'ethnologie et le Centre de documentation sociale de l'ENS recoivent chacun 3000 dollars, l'École coloniale de Paris 1000 , et l'Institut de géographie alpine de l'université de Grenoble $800^{75}$.

\section{La politique de recherche publique et les sciences sociales}

Si ces négociations commencent à se concrétiser à partir de 1932, c'est aussi en raison de la difficulté rencontrée par les sciences sociales à trouver leur place dans le dispositif de recherche publique alors en formation dans le cadre de la mobilisation scientifique. Dans ces conditions, les propositions de la fondation Rockefeller vont constituer pour Bouglé et Charléty une alternative inespérée.

Dans la genèse de la politique de recherche française ${ }^{76}$, l'année 1930 marque une étape décisive, avec la création de la Caisse Nationale des Sciences, dont les Français ont omis de signaler l'existence à leurs interlocuteurs américains au cours des discussions. La naissance de la CNS marque à la fois le début d'une politique nationale de la recherche pour les sciences humaines et sociales, mais aussi la séparation institutionnelle et intellectuelle de ces disciplines avec les Humanités ${ }^{77}$. En effet, à côté de la CNS est également créée une Caisse nationale des lettres $(\mathrm{CNL})$. Or, un certain nombre de disciplines qui, dans l'organisation universitaire, appartiennent aux facultés des lettres et de droit, ne sont pas intégrées dans la CNL mais bien dans la CNS, qui comprend deux sections : les sciences mathématiques et expérimentales d'une part, les sciences humaines de l'autre. A l'intérieur de cette dernière, coexistent cinq sous-sections: sciences philosophiques; sciences historiques, archéologiques et géographiques; sciences philologiques; sciences juridiques; sciences sociales. La section est pilotée par un comité technique divisé en cinq sous-comités correspondant aux sous-sections. Rist et Mauss font partie du

\footnotetext{
${ }^{73}$ Tracy Kittredge, Social Sciences in France, 30 juin 1932, RF 2/500S/557/3771.

${ }^{74}$ Elle a également, en janvier 1931, accordé 25000 dollars pour cinq ans à l'Institut international de Droit public, mais ce financement n'entre pas dans son programme français : créé en 1926, c'est un organisme indépendant de l'université française, quoique situé dans les locaux de la faculté de Droit et dirigé par Gaston Jèze et Boris Mirkine-Guetzévitch ; il est composé de chercheurs européens et américains (RF Minutes, 14 janvier 1931, 1.1/500/21/218).

${ }_{70}^{75}$ Formulaire d'attribution de bourse, 5 août 1932, RF 1.1/500S/23/233.

${ }^{76}$ Picard Jean-François, La république des savants: la recherche française et le CNRS, Paris, Flammarion, 1990.

77 Olivier Dumoulin, "Les sciences humaines et la préhistoire du CNRS », Revue française de sociologie, XXVI-2, 1985, p. 362.
} 
sous-comité des sciences sociales, Charléty de celui des sciences historiques ${ }^{78}$, tandis que Bouglé siège hors section, en tant que directeur adjoint de l'ENS.

La CNS est l'un des lieux où Bouglé tente de concrétiser son projet de coordination des sciences sociales. Mais celui-ci se heurte à plusieurs obstacles: d'une part le budget de la CNS accorde la part du lion à la section des sciences mathématiques et expérimentales, qui obtient en 1932, 4,14 millions de francs sur un total de 4,5 millions accordés aux allocations de recherches. D'autre part, la section des sciences humaines est dominée par les sciences historiques, archéologiques et géographiques, nettement mieux dotées que les autres. L'ambition coordinatrice de la sociologie se heurte ici à la montée en puissance de l'histoire, comme en témoigne l'appellation " sciences humaines " de la deuxième section : les " sciences sociales ॥ ne sont qu'une sous-section, autant dire une sous-préfecture, d'un ensemble dominé par l'histoire. Et surtout, à partir de 1934, la position des " sciences sociales ", déjà précaire au sein de la CNS, se dégrade encore : malgré la présence de Charléty, Rist, Mauss et Bouglé au Conseil Supérieur de la Recherche Scientifique (CSRS) créé en avril $1933^{79}$ pour piloter la recherche française, la rationalisation budgétaire imposée par la crise économique va s'effectuer à leur détriment: en 1933 est créée également une Commission des offices dont les conclusions rendues en novembre 1934 proposent la fusion de la Caisse des recherches scientifiques (CRS) créée en 1901 et de la CNS en un seul organisme, afin "d'assurer une meilleure coordination des services de recherche scientifique ${ }^{80}$ ": cette fusion se traduit par la création de la Caisse Nationale des Recherches Scientifiques (CNRS), effective au printemps 1935 et officialisée par le décret du 30 octobre $1935^{81}$. Dans la nouvelle organisation, la section « sciences humaines " ne dispose plus que de quatre sous-sections: sciences historiques et géographiques; sciences philologiques; sciences philosophiques; sciences juridiques et sociales $^{82}$. La sociologie, fusionnée avec le droit, est la principale victime de cette perte de visibilité intellectuelle et de la suppression d'une ligne budgétaire. Dans les débats qui ont lieu entre l'automne 1934 et le printemps 1935, Bouglé, présent notamment à la réunion du 27 novembre 1934 qui décide la création de la $\mathrm{CNRS}^{83}$, n'est pas parvenu à enrayer la dégradation de la position des sciences sociales ${ }^{84}$.

II va alors accentuer son investissement dans le projet franco-rockefellerien qui est monté en puissance entre-temps : en effet, après avoir renouvelé ses subventions aux instituts sus-cités en 1933, la Rockefeller les transforme en 1934 en un Fluid research fund ${ }^{85}$ dans le but avoué d' "élargir le cadre des recherches ${ }^{86}$ "; cette formule créée en 1929 par la fondation consiste en une dotation pluriannuelle laissant l'organisme récipiendaire répartir lui-même les crédits de recherche;

\footnotetext{
${ }^{78}$ Caisse Nationale des Sciences (ci-après CNS), rapport annuel 1932, AN F17/17458.

${ }^{79}$ liste des membres du CSRS, F17/17465. Charléty, Bouglé et Mauss avaient signé la pétition lancée en novembre 1932 par Jean Perrin (AN F17/17463).

${ }^{80}$ Conclusions adoptées par la Commission des offices dans sa séance du 27 novembre 1934, AN $61 \mathrm{AJ} / 102$.

${ }^{81}$ Jean-François Picard, «La création du CNRS », Revue pour l'histoire du CNRS, n¹, 1999, pp. 5066.

${ }^{82}$ Arrêté du 10 juillet 1936, AN F17/17464.

${ }^{83}$ Le compte rendu de cette réunion se trouve dans ses archives personnelles (voir note 81).

${ }^{84}$ Le déséquilibre budgétaire entre sciences mathématiques et expérimentales et sciences humaines perdurera jusqu'à la fin des années trente (CNS, rapport 1932 et brouillon du rapport 1934, AN F17/17458. Voir aussi Commission des finances de la CNRS, séance du 6 juin 1939, AN F17/17465).

${ }^{85}$ Rapport concernant la première année d'activité du CURS, 30 octobre 1935, RF 1.1/500S/24/243.

${ }^{86}$ Kittredge à Charléty, 4 juillet 1934, AN 61AJ/100.
} 
destinée d'abord aux universités américaines, elle a été étendue en 1933 à l'Europe, et en 1934, trois établissements en bénéficient pour les sciences sociales : la London School of Economics, (34 000 dollars) l'université de Stockholm (30 000) et l'Université de Paris $(21000)^{87}$. La condition sine qua none de l'octroi de ce fond étant la création d'une structure assurant la répartition de l'argent et officialisant le lien entre les sciences sociales et l'université, les directeurs des trois principaux instituts subventionnés depuis 1932 (Bouglé, Mauss et Henri-Léon Lévy-Ullmann pour l'Institut de droit comparé) décident en juin 1934 de créer un organisme ${ }^{88}$ intitulé Conseil Universitaire de la Recherche Sociale (CURS), auquel viendront s'agréger dans les mois suivants d'autres instituts. Tracy Kittredge présente alors à Charléty les conditions qui doivent être remplies pour obtenir la subvention : les recherches doivent être collectives et coordonnées, comme dans les sciences expérimentales ; elles doivent favoriser " une liaison intime et continue entre le monde académique et les personnalités qui ont la responsabilité de tâches administratives dans le domaine politique et social »; la subvention doit être utilisée pour payer salaires et bourses de voyages, et pour le «développement de méthodes et de moyens spéciaux d'enquêtes, tels que questionnaires et formules d'investigation ainsi que tout l'outillage propre à assurer l'obtention et l'analyse des faits empiriques ${ }^{89}$ ".

En octobre 1934, alors que les grandes lignes de la réorganisation du dispositif de recherche français filtrent sans doute déjà dans les couloirs du Ministère de l'éducation nationale, Bouglé saisit la perche tendue en juin par Kittredge et rédige un programme de travail collectif destiné à utiliser le Fluid research fund ${ }^{90}$, que les trustees de la fondation approuvent en décembre ${ }^{91}$. Alors qu'il ne semble guère assister au printemps 1935 aux réunions du conseil d'administration de la CNRS naissante dans laquelle les sciences sociales sont marginalisées ${ }^{92}$, Bouglé est au contraire pleinement impliqué dans la formalisation du dispositif franco-rockefellerien mis en place au même moment : au mois de juin 1935, la composition définitive du CURS est arrêtée et la Rockefeller reconduit le Fluid research fund pour cinq ans ${ }^{93}$, ouvrant ainsi la voie à la réalisation d'un vrai programme de recherches. Le CURS comprend alors sept organismes, en majorité des instituts d'université :

-Le Centre de documentation sociale (CDS) de l'ENS, créé en 1920 avec un financement du banquier Albert Kahn ${ }^{94}$, et dirigé par Célestin Bouglé depuis ses débuts $^{95}$ jusqu'à la mort de celui-ci en $1940^{96}$. Le financement Rockefeller va prendre le relais de celui de Kahn, ruiné par la crise de 1929.

-L'Institut d'ethnologie (IE) de l'université de Paris fondé par Marcel Mauss et Lucien Lévy Brühl en $1925^{97}$.

-L'Institut de Droit comparé (IDC), fondé en juillet $1931^{98}$, et auquel la subvention Rockefeller permet de démarrer son activité.

\footnotetext{
${ }^{87}$ Fluid research funds, Report of Committee on Appraisal, 11 décembre 1934, RF 3/900/24/189.

${ }^{88}$ Charléty à Kittredge, 27 juin 1934, CAC 20010498/111.

${ }^{89}$ Kittredge, 15 juin 1934, CAC 20010498/111.

${ }^{90}$ Kittredge à Charléty, 7 février 1935, Bouglé à Charléty, 26 février 1935 -35, CAC 20010498/111.

${ }^{91}$ Fluid research funds, Report of Committee on Appraisal, 11 décembre 1934, RF 3/900/24/189.

92 Un indice de ce manque d'assiduité inhabituel chez Bouglé : dans les trois seuls procès verbaux de séance, datés de mars, conservés dans les archives de la CNRS pour l'année 1935, Bouglé n'apparaît pas parmi les membres présents (AN F17/17464).

${ }^{93}$ Rapport concernant la première année d'activité du CURS, 30 octobre 1935, RF 1.1/500S/24/243.

94 Sophie Coeuré, "Les centres de documentation sociale, 1920-1940 », in Pascal Ory (dir.), Albert Kahn (1860-1940), Réalité d'une utopie, Boulogne, Musée Albert Kahn, 1995, pp. 201-210.

${ }^{95}$ Note biographique, 61AJ/104.

${ }^{96}$ Dossier de Célestin Bouglé, AN AJ16/5885.

${ }^{97}$ Emmanuelle Sibeud, op. cit., p. 266.
} 
-L'Institut français de Damas (IFD), créé en 1922 pour favoriser « l'affirmation de la présence culturelle française dans les territoires sous mandat [de la SDN] ${ }^{99}$ ", et consacré initialement à l'archéologie, la linguistique et la littérature, devient en 1930 un institut dédié à la recherche scientifique ; l'année suivante, il obtient à cet effet le double patronage de l'Institut d'ethnologie et de l'université de Paris.

-L'Institut de géographie (IG), créé en $1926^{100}$.

-Le Comité français pour l'étude scientifique des populations (CFESP), dirigé par l'économiste et parlementaire Adolphe Landry, qui constitue la branche française de l'Union internationale pour l'étude scientifique des problèmes de population créée en $1928^{101}$

-La Commission des recherches collectives (CRC) de l'Encyclopédie française, fondée en $1934^{102}$ et dirigée par Lucien Febvre, René Maunier, Albert Demangeon et Paul Rivet.

Rattaché à l'Université et Paris et placé sous la responsabilité du recteur qui administre et répartit son budget, le CURS recevra entre 1934 et 1940166000 dollars qui représentent l'intégralité de ses ressources. Par ailleurs, le compromis florentin entériné au printemps 1931 entre les objectifs de la fondation et ceux des protagonistes français en présence se traduit par le soutien parallèle de la fondation à deux instituts indépendants de l'université : I'Institut scientifique de recherches économiques et sociales (ISRES) de Charles Rist, créé officiellement en décembre 1933 et doté d'emblée de 350000 dollars pour une période de 7 ans (1933-1940) ${ }^{103}$; le Centre d'étude de politique étrangère (CEPE), créé en février 1935. L'idée de créer une structure spécialisée dans l'étude des relations internationales, qui n'apparaît qu'en 1932 dans les négociations ${ }^{104}$, a été portée par Bouglé et Charléty ; la fondation lui accordera 170000 dollars jusqu'en 1940, couvrant la grande majorité de ses frais de fonctionnement ${ }^{105}$.

L'examen rapide du financement américain permet une première évaluation de la place prise par ce dispositif dans le paysage français des sciences sociales. En 1932, le financement Rockefeller est de 10800 dollars (162 000 francs) pour cinq organismes, soit un tiers de la somme allouée par la CNS aux allocations de recherche pour l'ensemble des sciences humaines. En 1935, il monte à 120000 dollars (25 000 pour le CURS, 50000 pour l'ISRES, 34000 pour le CEPE, et environ 14000 dollars pour 7 fellowships), soit 1,8 millions de francs, c'est-à-dire pratiquement le double du budget total de la CNS pour les allocations en sciences humaines. Au total, si l'on additionne les subventions aux trois grandes structures (CURS, ISRES, CEPE) et les fellowships, on peut estimer le total de la subvention Rockefeller à 700000 dollars entre 1934 et 1940 , soit 16,5 millions de francs ${ }^{106}$, alors que dans le même temps, la CNS aura accordé environ 11 millions de francs

\footnotetext{
${ }^{98}$ Charléty à Van Sickle, 28 septembre 1931, CAC 20010498/111.

${ }^{99}$ Christophe Charle, «Enseignement supérieur et expansion internationale (1870-1930). Des instituts pour un nouvel empire ? » in : Johan Heilbron, Rémi Lenoir \& Gisèle Sapiro (dir.), op. cit., p. 335.

${ }_{100}$ Décret du 8 mai 1926, CAC 20010498/62.

101 Paul-André Rosental, L'intelligence démographique. Science et politique des populations en France (1930-1960), Paris, Odile Jacob, 2003, p. 24.

${ }^{102}$ André Varagnac, "Chronique du Secrétariat », Revue de synthèse, décembre 1935.

${ }^{103}$ Ludovic Tournès, "L'institut Scientifique de Recherches Economiques et Sociales et les débuts de l'expertise économique en France (1933-1940)", Genèses. Sciences sociales et histoire, $n^{\circ} 65$, décembre 2006, pp. 49-70.

${ }^{104}$ Tracy Kittredge, 1 décembre 1933, RF 1.1/500S/20/204.

105 Rapport financier 1936-1937 (RF 1.1/500S/21/210), 1937-1938 (AN 61AJ/100), 1939-1940 (RF $1.1 / 500 \mathrm{~S} / 21 / 211)$.

${ }^{106}$ Le dollar vaut 15,87 francs jusqu'à la dévaluation de 1936, puis 29,27 francs après cette date.
} 
d'allocations de recherches aux sciences humaines ${ }^{107}$. Même en estimant au maximum à 5 millions de francs sur l'ensemble de la période 1934-1940 les autres types d'aides données par la CRS puis par la CNRS (subventions aux publications, achat de matériel) ${ }^{108}$, il apparaît que le financement Rockefeller pour les sciences sociales au cours de la deuxième moitié des années trente est au moins équivalent, et très probablement supérieur, à celui du dispositif de recherche publique français. On ajoutera que, dans le budget d'au moins six des neufs organismes financés, l'argent Rockefeller représente entre un tiers (IE ${ }^{109}$, IDC en $1935^{110}$ ) et la quasitotalité (CEPE, IDC en $1936^{111}$ ), voire la totalité (CDS à partir de $1933^{112}$, ISRES). L'IFD oscille vraisemblablement entre ces deux pôles : mal doté depuis ses débuts, c'est certainement sur l'intervention de Charléty que son budget est rattaché à partir de 1935 à l'université de Paris ${ }^{113}$, ce qui lui permet de recevoir les subventions du CURS. La subvention Rockefeller étant destinée uniquement aux activités de recherches, quasi-absentes des subventions ministérielles couvrant essentiellement les salaires et les frais de fonctionnement, la grande majorité du travail scientifique de ces instituts n'aurait pu être réalisée sans ces crédits.

\section{UN NOUVEAU PARADIGME : LA RECHERCHE EXPERIMENTALE}

Si l'on se penche maintenant sur la production intellectuelle mise en œuvre dans cet ensemble d'instituts, une constatation s'impose : la structure franco-rockefellerienne occupe une place incontournable dans le paysage des sciences sociales de la décennie 1930. Du point de vue quantitatif d'abord: trois périodiques sont créés grâce à la subvention : L'activité économique, trimestriel publié par l'ISRES à partir de 1935 ; les Annales Sociologiques (1934), revue annuelle réalisée au CDS; Politique étrangère (1936), trimestriel publié par le CEPE. Quant aux livres, le CDS en publie au moins 13 entre 1934 et 1940 (principalement chez l'éditeur Alcan), I'ISRES 15 (le plus souvent chez Sirey) et le CEPE 29 (aux éditions Hartmann), I'IFD 5, dont 4 en collaboration avec le CEPE, I'IDC 7, I'IE 14 (dont 12 dans la collection des Travaux et mémoires de l'Institut d'ethnologie) soit au total au moins 80 ouvrages, auxquels on ajoutera les articles publiés dans d'autres revues. Mais audelà de l'aspect quantitatif, cette production possède une unité intellectuelle fondée sur la mise en œuvre du paradigme de la recherche expérimentale, qui témoigne de la cohérence du projet et du rôle important de cet ensemble d'instituts dans la cristallisation d'un champ des sciences sociales en France.

\footnotetext{
${ }^{107} 432000$ francs en 1932, 1 million par an entre 1933 et 1936, puis 1,9 millions par an entre 1937 et 1940. (AN F17/17458, 17459 et 17465).

${ }^{108}$ Par exemple, en 1939, la CNRS accorde 16500 francs aux sciences humaines au titre des achats de matériel pour la recherche, et 658000 francs pour les subventions aux publications, deux rubriques qui correspondent aux anciennes fonctions de la CRS avant sa fusion avec la CNS en 1935 (AN F17/17465).

${ }_{109}$ Exercice 1932, CAC 20010498/70.

${ }_{110}$ Comptes pour 1935, CAC 20010498/111.

${ }_{112}^{111}$ Exercice 1936-37, CAC 20010498/111.

112 Budget pour 1933, 61AJ/97; CURS, Rapport 1935-36, 1936-1937 et 1937-1938, RF 1.1/500S/24/243. A partir de 1934, le reliquat de la donation Kahn n'apparaît plus dans les comptes.

${ }^{113}$ Renaud Avez, L'institut français de Damas au palais Azem (1922-1946), Damas, 1993, p. 84-85 et p. 100.
} 


\section{La sociologie au cœur du dispositif}

Alors que la CNS est articulée autour de l'histoire, le CURS a comme pôle dominant la sociologie. Trois des quatre instituts les mieux dotés se situent dans l'orbite durkheimienne (le CDS, l'IE, L'IFD) ; la bonne dotation de I'IDC ainsi que la forte présence des juristes au sein du conseil d'administration du CURS (6 sur 16 membres $^{114}$ ) suggèrent que Bouglé a fait alliance avec les sciences juridiques, sacrifiées avec les science sociales sur l'autel de la rationalisation budgétaire en 1935 ; quant à l'histoire (à travers la CRC) et la géographie, elles sont nettement moins bien dotées, et ne sont représentées au Conseil que par Albert Demangeon et Henri Hauser. On a pu s'interroger sur le fait que la faible présence de l'histoire était le résultat d'une exigence de la Rockefeller ${ }^{115}$. Cette vision doit être nuancée. D'abord parce que l'histoire fait partie des disciplines envisagées par le programme du LSRM dès 1924, même si elle apparaît derrière l'économie, la sociologie et la science politique ${ }^{116}$; Par ailleurs, la Rockefeller subventionne dans les années suivantes des programmes où l'histoire est importante, et dont le plus connu est le comité international d'histoire des prix ${ }^{117}$. En France, le premier financement collectif qu'elle accorde, avant même les subventions de 1932, concerne huit historiens français, pour la plupart professeurs de lycée, à qui elle accorde 6000 dollars en mars 1929 pour des congés sabbatiques et des voyages à l'étranger destinées à avancer leurs recherches ${ }^{118}$. On peut également signaler les fellowships accordées à deux historiens, Désiré Pasquet en 1925 (pour la réalisation de son Histoire politique et sociale du peuple américain publiée entre 1925 et 1931) et Victor-Lucien Tapié en 1933 (pour compléter la documentation de ses thèses de doctorat ${ }^{119}$ ). La marginalisation de l'histoire dans le CURS est donc au moins autant, sinon plus, due à Bouglé qu'à la Rockefeller, et constitue à bien des égards une réponse à la marginalisation de la sociologie dans la CNS.

La sociologie joue un rôle central dans l'adoption par la structure francorockefellerienne d'une posture épistémologique fondée sur la méthode inductive et expérimentale. Celle-ci fait partie du cahier des charges présenté par Tracy Kittredge et concrétise le projet rockefellerien de " casser [le] patron [pattern] académique » de la tradition spéculative et d'établir un nouveau " moule ${ }^{120}$ " [mold] intellectuel dans les sciences sociales. Déjà bien installée dans les sciences sociales américaines, en particulier dans la sociologie ${ }^{121}$, cette posture est relayée par Mauss et Bouglé ${ }^{122}$ qui mettent en avant l'exemple américain dans la réalisation de programmes collectifs fondés sur les recherches empiriques. Ces méthodes ne sont pas pour autant une

\footnotetext{
${ }^{114}$ Rapport concernant la première année d'activité du CURS, 30 octobre 1935, RF 1.1/500S/24/243.

${ }^{115}$ Brigitte Mazon, op. cit., p. 67.

${ }^{116}$ Memorandum on Fellowships in the Social Sciences, 14 avril 1924, LSRM I/1/11.

117 Olivier Dumoulin, «Aux origines de l'histoire des prix », Annales. Histoire sciences sociales, 45-2, 1990, pp. 507-522.

${ }_{118}$ RF Minutes, 8 mars 1929, 1.1/500/18/181.

${ }^{119}$ La politique étrangère de la France et le début de la guerre de trente ans (1616-1621), et Une église tchèque au XVe siècle : l'unité des frères, Paris, 1934. Tapié écrira en 1936 dans le cadre des travaux du CEPE un ouvrage sur Le pays de Teschen et les rapports entre la pologne et la tchécoslovaquie.

${ }^{120}$ A brief summary of the conference of trustees and officers at Princeton, octobre 1930, RF $3 / 900 / 22 / 166$.

${ }_{121}$ Jean-Michel Chapoulie, La tradition sociologique de Chicago (1892-1961), Paris, Le Seuil, 2001, pp. $146 \mathrm{sq}$.

${ }^{22}$ Annales Sociologiques, série A, fascicule 1, 1934, p. 96.
} 
création américaine : les sociologues leplaysiens ${ }^{123}$ ou les géographes ${ }^{124}$, pour ne citer qu'eux, pratiquent l'enquête de terrain depuis longtemps. Mais celle-ci reste marginale jusqu'aux années trente, et le dispositif franco-rockefellerien va largement contribuer à la promouvoir au rang d'outil essentiel d'un travail scientifique sur les faits sociaux. La montée en puissance de cette approche empirique et quantitativiste est l'un des symptômes les plus importants de la cristallisation d'une collectivité des sciences sociales marquée par une volonté commune de rompre avec l'étude purement livresque des faits sociaux.

\section{Des programmes collectifs}

Dans cet ensemble composé de I'ISRES, du CEPE et du CURS, ce dernier est le principal vecteur de l'ambition de "coordination intellectuelle ${ }^{125}$ ", à travers son programme ratifié en juin $1935^{126}$. Alors que la CNS, puis la CNRS, restent des " caisses » dont n'émane aucun projet, le CURS s'organise autour d'un ensemble de thématiques. Son programme primitif comprend dix rubriques: les «questions sociales et la psychologie ouvrière », et en particulier «les conséquences du machinisme "; "les transformations politiques [...] des états modernes »; les «tendances nationales" et notamment la «psychologie des peuples et les mouvements d'opinion »; les «populations primitives »; "la colonisation » et "l'influence de la civilisation sur le genre de vie des peuples colonisés "; "le folklore rural »; les « questions démographiques »; les « recherches sur la situation et les droits de la femme "; "l'organisation des consommateurs »; le « recensement des centres d'études sociales et spécialement d'études internationales en France $^{127}$ ». En 1935, elles sont regroupées en quatre ensembles correspondant largement aux divisions disciplinaires ${ }^{128}$ : des «travaux de sociologie » réalisés essentiellement au CDS ; des recherches sur les «populations primitives et les questions coloniales ॥ réalisées par l'IE et l'IFD, auxquelles s'ajoute l'enquête du CFESP sur la démographie des colonies françaises; les "enquêtes juridiques " de l'IDC ; les " enquêtes rurales » de l'IG et de la CRC.

Ce plan de travail prévoit la mise en œuvre de recherches collectives, chaque rubrique étant coordonnée par un ou plusieurs " directeurs d'études "129 " membres du Conseil d'administration, sous la direction desquels travaillent des jeunes chercheurs, mais aussi des collaborateurs occasionnels, souvent correspondants locaux de sociétés savantes. Cette nouvelle pratique de recherche n'est pas spécifique au dispositif Rockefeller (elle est aussi à l'origine de la création de la $\mathrm{CRC}$ ), mais il est l'un des lieux importants de son développement. Elle prend des formes diverses : au CDS, des réunions hebdomadaires autour des travaux en cours

\footnotetext{
${ }^{123}$ Antoine Savoye, Les débuts de la sociologie empirique : études socio-historiques, 1830-1930, Paris, Klincksieck, 1994.

124 Marie-Claire Robic, «Interroger le paysage? L'enquête de terrain. Sa signification dans la géographie humaine moderne (1900-1950) », in Claude Blanckaert (dir.), Le terrain des sciences humaines (XVIIle-XXe siècle), Paris, L'Harmattan, 1996, pp. 357-388.

${ }^{125}$ Paul Vaucher, Le conseil universitaire de la recherche sociale, n.d. (1937), AN 61AJ/100.

${ }^{126}$ Rapport concernant la première année d'activité du CURS, 30 octobre 1935, RF 1.1/500S/24/243. Voir aussi procès verbal du 9 juillet 1936, CAC 20010498/111.

${ }_{128}^{127}$ Programme des travaux en cours, 26 février 1935, 1.1/500/24/241.

${ }^{128}$ La dénomination des grands axes ne variera pratiquement plus jusqu'en 1939 (Rapports du CURS pour les années 1935 à 1939, 1.1/500/24/241, 242 et 243.

${ }^{129}$ Programme, 26 février 1935, 1.1/500/24/241.
} 
des membres aboutissent à la publication de trois volumes collectifs en 1936, 1937 et $1938^{130}$. A l'IDC est inaugurée à la rentrée 1933 une pratique similaire qui constitue une «innovation [...] à la fois pédagogique et scientifique ${ }^{131}$ » chez les juristes et qui trouve son débouché dans la publication des Travaux pratiques de l'Institut de Droit comparé. Mais surtout, tous les organismes du dispositif entreprennent des enquêtes collectives qui constituent une nouveauté des années trente dans le paysage des sciences sociales : I'IDC lance en 1934 une recherche sur la condition juridique de la femme contemporaine ${ }^{132}$, puis une enquête sur le régime juridique du chèque dans le monde ${ }^{133}$. A l'IG sont réalisées entre 1935 et 1938 trois enquêtes coordonnées par Albert Demangeon, qui reprend un projet ébauché dès 1920 mais laissé en jachère, sans doute faute de moyens financiers ${ }^{134}$ : la première porte sur les étrangers dans l'agriculture française, et utilise le concours d'instituteurs, de professeurs de lycées et d'universitaires ; elle est réalisée à partir d'un questionnaire envoyée à trois mille personnes ${ }^{135}$ et de statistiques issues des préfectures départementales dont Charléty a obtenu du ministère de l'Intérieur l'autorisation d'utilisation ${ }^{136}$. La deuxième porte sur l'habitat rural ${ }^{137}$ et la troisième sur les structures agraires ${ }^{138}$. La CRC reçoit également les subsides du CURS pour trois enquêtes (les usages de moisson et de fenaison et les feux traditionnels ; l'évolution récente de la forge de village ; l'alimentation populaire traditionnelle ${ }^{139}$ ), également fondées sur des questionnaires ${ }^{140}$ et menées par des «nombreuses équipes d'observateurs spécialisés se déplaçant fréquemment selon un itinéraire méthodique », ou avec un "réseau permanent d'informateurs ${ }^{141}$ ". Le CFESP réalise une recherche sur la démographie des colonies françaises, partie intégrante d'un vaste projet d'étude des populations présenté lors du premier congrès

\footnotetext{
130 Inventaires I, II, III, Paris, Alcan.

131 Université de Paris. Faculté de Droit. Institut de Droit comparé, Travaux pratiques de Droit privé comparé, vol. 1, Paris, 1935.

132 Marc Ancel (dir.), La condition de la femme dans la société contemporaine, Paris, Sirey, 1938.

133 Joseph Hamel et Marc Ancel (dir.), La convention de Genève sur l'unification du droit du chèque, Travaux et mémoires de l'Institut de droit comparé de l'université de Paris, Sirey, 1937.

${ }^{134}$ Albert Demangeon, "Trois questionnaires et trois enquêtes de géographie humaine », Annales de géographie, septembre 1936, pp. 512-518.

${ }^{135}$ CURS, Documents pour servir à l'étude des étrangers dans l'agriculture française, Paris, Hermann, 1939.

${ }^{136}$ Ministère de l'intérieur. Direction générale de la sûreté nationale à Charléty, 25 février 1937, CAC 20010498/111.

${ }^{137}$ Albert Demangeon et Jean Gottmann, La maison rurale en France, Paris, Denoël, 1937.

138 Elle sera publiée en 1964 par l'élève de Demangeon Jean Gottmann, Documents pour servir à l'étude de la structure agraire dans la moitié occidentale de la France. Rapport de l'enquête dirigée par Albert demangeon en 1936-1940, Paris, Armand Colin.

${ }^{139}$ Lucien Febvre, "Une enquête : la forge de village ", Annales d'histoire économique et sociale, novembre 1935, pp. 603-614 ; Id, «Répartition géographique des fonds de cuisine en France », Travaux du premier congrès international de folklore, Paris, 23-28 août 1937, Paris, MNATP pp. 122130, réédité dans dans Tiphaine Barthélémy et Florence Weber (eds), Les campagnes à livre ouvert, Regards sur la France rurale des années 30, Paris, Presses de l'ENS/ éditions de l'EHESS, 1989 ; André Varagnac, "La nourriture, substance sacrée », Annales d'histoire sociale, 3-12, 1941, pp. 2230 , réédité dans Barthélémy et Weber, op cit.

${ }^{140}$ Bertrand Muller, « Entre science et culture », in Jacqueline Pluet-Despatin et Gilles Candar (dir.), Lucien Febvre et l'Encyclopédie française, Cahiers Jaurès, n¹63-164, janvier-juin 2002, p. 49.

141 André Varagnac, "Une coopérative de travail scientifique: la Commission des recherches collectives du comité de l'Encyclopédie française ", Annales d'histoire économique et sociale, mai 1935, p. 303.
} 
international de la population en $1937^{142}$. Enfin, I'ISRES lance au printemps 1935 une grande enquête sur le chômage ${ }^{143}$, et le CEPE crée entre 1935 et 1939 quatorze groupes d'études thématiques. On n'oubliera pas le projet d'enquête entrepris en 1938 par le CDS sur les classes moyennes, dont l'ambition est de réaliser une synthèse entre l'accumulation de données quantitatives dont la sociologie américaine donne l'exemple, et une réflexion globale sur la notion de groupe social inspirée de la démarche durkheimienne ${ }^{144}$, un projet que la guerre empêchera de mener à son terme.

Si le programme du CURS correspond aux frontières disciplinaires, son ambition est également de favoriser des passerelles pour dépasser « la division traditionnelle en France entre faculté de droit et faculté des lettres ${ }^{145}$ ». Charléty et Bouglé, membres des conseils d'administration du CURS, de l'ISRES et du CEPE, jouent le rôle de trait d'union entre eux : c'est Bouglé qui insiste auprès de ses collègues de l'ISRES pour que des enquêtes réunissant sociologues et économistes soit entreprises, suggérant l'idée de l'enquête sur le chômage ; c'est lui également qui suscite la réalisation d'un travail commun entre l'IFD et le Groupe d'études islamiques du CEPE en 1935 ; c'est enfin lui qui crée au CEPE un groupe d'études sur les sciences sociales dont le rapport collectif publié en $1937^{146}$ rassemble, parmi les contributeurs, des membres du CDS (Bouglé, Raymond Aron), de l'ISRES (Robert Marjolin, Henri Lemaître) et du CEPE (Jacques Lambert).

\section{Le CDS et le renouveau de la sociologie}

Grâce au financement Rockefeller, le CDS peut, à partir de 1932, développer les recherches empiriques que Bouglé appelle de ses vœux. Les crédits américains servent d'abord à financer jusqu'en 1939 deux postes d'assistants ${ }^{147}$. Les bénéficiaires sont tous des normaliens agrégés de philosophie choisis par Bouglé. Le premier poste est celui de secrétaire administratif du CDS : de 1932 à 1934, il est assuré par Philippe Schwob : celui-ci a obtenu une fellowship en 1930 pour un séjour de deux ans aux Etats-Unis au cours duquel il a recueilli le matériau pour sa thèse d'économie, rédigée au cours de sa période d'assistanat ${ }^{148}$. Puis Raymond Aron lui succède de 1934 à 1939 : parti en 1930 en Allemagne où il est resté trois ans, il est ensuite nommé professeur au lycée du Havre avant d'intégrer le CDS. Son intérêt pour les sciences sociales, amorcé lors de son séjour allemand ${ }^{149}$, s'accentue à partir du moment où il travaille au CDS, réalisant des analyses sur les problèmes

\footnotetext{
142 Congrès international de la population, Paris, 1937, volumes 1 à 8 , Paris, Hermann, 1938. Le volume 6 est consacré à la démographie des possessions françaises.

${ }^{143}$ Ludovic Tournès, "Le durkheimisme face à ses contradictions: L'enquête sur le chômage de l'Institut Scientifique de Recherches Economiques et Sociales (1935-1937) », Revue Française de Sociologie, 47-3, juillet-septembre 2006, pp. 537-559.

${ }^{144}$ Henri Mougin, "Projet d'enquête sur les classes moyennes ", in : Inventaires III, Paris, 1938, p. 294.

${ }_{145}$ Paul Vaucher, Le conseil universitaire de la recherche sociale, n.d. (1937), AN 61AJ/100.

${ }_{146}$ CEPE, Les sciences sociales en France. Enseignement et recherche, Paris, Hartmann, 1937.

${ }^{147}$ CAC 20010498/111 et 112.

${ }_{148}$ Philippe Schwob, Les investment trust aux Etats-Unis, thèse de doctorat, Paris, Sirey, 1934.

149 Nicolas Baverez, Raymond Aron, un moraliste au temps des idéologies, Paris, Flammarion, 1993, pp. 73 sq.
} 
actuels $^{150}$ et des travaux sur la sociologie ${ }^{151}$ tout en travaillant à sa thèse de philosophie soutenue en $1938^{152}$. Mais Aron est peu féru de méthode expérimentale, à laquelle il préfère l'analyse qualitative, et ne s'inscrit que marginalement dans le processus d'affirmation du paradigme expérimental dans les sciences sociales. Le deuxième poste d'assistant financé par les fonds Rockefeller est celui de répétiteuragrégé pour les élèves normaliens. De 1932 à 1935, il est occupé par Georges Friedmann: venu à la sociologie par le marxisme ${ }^{153}$, il engage pendant ses trois années au CDS une réflexion sur le machinisme appuyée sur un travail de terrain effectué lors de deux séjours en URSS en 1932 et $1933^{154}$. De 1935 à 1938, c'est Raymond Polin qui lui succède ; ce dernier a réalisé en 1934 un travail sur les coopératives en Tchécoslovaquie fondé sur les statistiques de l'administration thécoslovaque collectées lors d'un séjour sur place ${ }^{155}$; Bouglé l'engage au CDS pour continuer dans cette direction tout en travaillant à sa thèse de philosophie soutenue en 1944. Enfin, de 1938 à 1940, la suite est prise par André Kaan. Outre ces deux postes d'assistants, la fondation accorde à Bouglé une fellowship pour Robert Marjolin, membre du CDS non normalien, qui part en 1932 pour un an à l'université de Yale. Ce jeune autodidacte qui se destinait à la philosophie arrive en plein début du New Deal, à l'étude duquel il consacrera une partie importante de son séjour ${ }^{156}$. Tous ces jeunes chercheurs sont également mobilisés pour la réalisation des Annales Sociologiques, nouvelle tribune de l'école durkheimienne, notamment pour réaliser les nombreux comptes rendus et notices bibliographiques ${ }^{157}$, poursuivant la tradition inaugurée en 1896 par l'Année sociologique. Une autre partie de la subvention Rockefeller est utilisée pour des aides ponctuelles à des membres du CDS : Entre 1935 et 1937, ce sont près d'une vingtaine de jeunes chercheurs, pas toujours issus de l'ENS, qui en bénéficieront ${ }^{158}$ : c'est le cas, entre autres exemples, de Stefan Wlocevski, qui a réalisé sous la direction de Bouglé en 1934 une enquête sur les italiens en France à partir de témoignages et de données d'origine administrative ${ }^{159}$, et qui, entre 1935 et 1937, mène un travail sur les ouvriers polonais $^{160}$ dans le cadre de l'enquête sur les étrangers dans l'agriculture (cf. supra).

\footnotetext{
150 «L'Allemagne: une révolution antiprolétarienne. Idéologie et réalité du national-socialisme », Inventaires I. La crise sociale et les idéologie nationales, Paris, Alcan, 1936, pp. 24-55; «Les rapports de la politique et de l'économie dans la doctrine marxiste », Inventaires II. L'économique et le politique, Paris, 1937, pp. 16-47 ; « Le concept de classe ॥, Inventaires III, Classes moyennes, Paris, Alcan, 1939, pp. 7-27; voir aussi «Réflexions sur les problèmes économiques français, Revue de métaphysique et de morale, octobre 1937, pp. 793-822.

${ }_{151} \mathrm{La}$ sociologie allemande contemporaine, Paris, Alcan, 1935 ; «La sociologie », CEPE, Les Sciences sociales en France, op. cit., pp. 13-48 (co-écrit avec Celestin Bouglé).

152 Introduction à la philosophie de l'histoire. Essai sur les limites de l'objectivité historique, Paris, NRF, 1938.

${ }^{153}$ Daniel Lindenberg, « Georges Friedmann et la Revue Marxiste », in Pierre Grémion et Françoise Piotet (dir.), Georges Friedmann, un sociologue dans le siècle (1902-1977), Paris, CNRS éditions, 2004 , p. 18.

154 Georges Friedmann, Problèmes du machinisme en URSS et dans les pays capitalistes, Paris, Editions Sociales internationales, 1934 ; voir l'article " Aspects du machinisme en URSS ».

155 Raymond Polin et J.G Charon, Les coopératives rurales et l'Etat en Tchécoslovaquie et en Roumanie, Paris, Alcan, 1934, p. 4.

${ }_{157}^{156}$ Les expériences Roosevelt, Paris, 1934.

157 Voir notamment les comptes rendus de Raymond Aron, André Kaan et Robert Marjolin et Raymond Polin dans la série A (sociologie générale, fascicules 1 à 4) et de Robert Marjolin dans la série $D$ (sociologie économique, fascicules 3 et 4 ).

${ }_{158}$ On trouve à ce sujet de nombreuses lettres dans AN 61AJ/97 et 61AJ/100.

159 Stefan Wlocevski, L'installation des Italiens en France, Paris, Alcan, 1934, p. 17.

160 Bouglé à Charléty, 22 décembre 1936, AN 61AJ/97.
} 
Enfin, le reste de la subvention sert acheter des livres et à financer les publications des ouvrages.

\section{Des ethnologues sur le terrain}

La recherche expérimentale est également à l'ordre du jour des travaux menés sur les pays colonisés par l'IFD et l'IE. Là encore, de telles recherches existent avant l'arrivée de la subvention Rockefeller, mais elle va leur donner une impulsion décisive. Le Commandant Robert Montagne, en poste au Maroc depuis 1918, a ainsi réalisé dans la deuxième moitié des années vingt une thèse de doctorat sur l'impact de la colonisation sur les berbères à partir d'enquêtes ethnographiques menées dans les villages de l'Atlas marocain ${ }^{161}$. Sa promotion à la tête de l'Institut français de Damas et l'importance du financement Rockefeller lui permettent de donner corps à un ambitieux projet d'étude en collaboration avec le groupe d'études islamique du CEPE, portant sur les transformations des sociétés colonisées, dont il trace le plan dans un article programmatique publié dans les Annales sociologiques en 1935, et dont la méthode repose sur "l'observation méthodique des faits ", l'établissement de "fiches $^{162}$ " et le dépouillement de la presse arabe. Ce programme se concrétise par des études collectives sur plusieurs pays arabes ${ }^{163}$, et trois ouvrages issu de colloques organisées par le CEPE et I'IFD à Paris en 1936, 1937 et $1938^{164}$, en collaboration avec l'Institut des études islamiques de l'université de Paris, combinant l'étude des questions historiques à celle des problèmes d'actualité. A ce projet collectif s'ajoutent des travaux individuels mené par les membres de l'IFD : ceux de Robert Montagne (9 articles dans Politique étrangère entre 1936 et 1940), mais aussi ceux du capitaine Albert de Boucheman, qui publie en 1934 une description des objets quotidiens des bédouins ${ }^{165}$ et réalise en 1935-36, avec l'aide du CURS, l'étude ethnographique approfondie d'une petite ville caravanière à travers laquelle il aborde le problème du passage du nomadisme au sédentarisme et «le bouleversement économique et social qui ruine le désert ${ }^{166}$ ". On ajoutera la thèse de doctorat de Jacques Weulersse sur le nomadisme en Syrie, commencée dans le cadre d'une mission patronnée par l'IE en $1932^{167}$ et poursuivie à l'IFD ${ }^{168}$.

Le travail de terrain est également en plein développement à l'IE. Si Mauss lui-même n'a jamais mené de recherches de terrain, il encourage dès la fin des années vingt ses élèves dans ce sens. D'abord consacré à l'enseignement, I'IE patronne les premières missions en 1928, en particulier celle de Marcel Griaule en Abyssinie, au cours de laquelle il met en œuvre la technique de l'observation directe. En 19311933, 20\% au moins du budget de la mission Dakar-Djibouti de Griaule vient de la

\footnotetext{
${ }^{161}$ Robert Montagne, Les Berbères et le Makhzen. Essai sur la transformation politique des Berbères sédentaires (groupe chleuh), thèse de doctorat ès lettres, université de Paris, 1930.

162 Robert Montagne, "L'évolution moderne des pays arabes », Annales sociologiques, Série A, fascicule 2, 1935, p. 66.

${ }_{163}$ Le groupe d'études de l'islam, L'égypte indépendante, Paris, Hartmann, 1938 ; Gabriel Bounoure, Louis Dubertret et Jacques Weulersse, Manuel de Géographie (Syrie, Liban et Proche Orient), Beyrouth, 1940.

${ }^{164}$ CEPE, Entretiens sur l'évolution des pays de civilisation arabe, Paris, Hartmann, 1936 (vol. I), 1937 (vol. II) et 1938 (vol III.

${ }^{65}$ Albert de Boucheman, Matériel de la vie bédouine recueilli dans le secret de Syrie (tribu des arabes Sba'a), Documents d'études orientales, tome III, IFD, 1934.

${ }^{166} \mathrm{Id}$, Une petite cité caravanière : Suhné, Documents d'études orientales, tome VI, IFD, 1937.

167 IE, rapport 1931-32, CAC 20010498/70.

168 Jacques Weulersse, Le pays des Alaouites, thèse de doctorat, Faculté des lettes de l'université de Paris, 1940
} 
subvention Rockefeller, sous la double forme d'une fellowship et de la subvention accordée à l'IE en $1932^{169}$. Cette enquête, qui « inaugure officiellement l'ère des grandes enquêtes de terrain de l'ethnologie française ${ }^{170}$ " et marque une étape importante dans la professionnalisation de l'ethnologie, relève également d'une logique quantitativiste : si elle n'utilise pas l'outil statistique, son résultat le plus visible est la « collection de 3500 objets pour le musée d'ethnographie du Trocadéro, 6000 photographies, 3500 mètres de film, 200 enregistrements sonores ${ }^{171}$ "; les objets recueillis font l'objet d'un «rigoureux protocole d'observation ${ }^{172}$ " matérialisé par " une fiche descriptive complète donnant tous renseignements sur la provenance, l'usage, la fabrication, etc., de l'objet ${ }^{173}$ ". La même logique fondée sur la collecte de faits ethnologiques est à l'œuvre dans les autres enquêtes de l'IE, dont trois au moins sont financées partiellement par les crédits Rockefeller, notamment sous la forme de fellowships accordées à des élèves de Mauss en compensation de son éviction de janvier 1931 : la première pour Georges Devereux sur les peuples Moï d'Indochine en 1932-1934 ; la deuxième pour Charles Le Cœur, parti en 1933 pour le Tibesti tchadien ${ }^{174}$ enquêter sur les populations locales en suivant la méthode des « monographies familiales librement inspirées de Le Play ${ }^{175}$ "; la troisième pour Denise Paulme en 1935, au cours de laquelle elle accompagne Marcel Griaule en pays Dogon puis réalise sa propre enquête qui donnera lieu à sa thèse de doctorat $^{176}$. On ajoutera que les subventions Rockefeller financent également une partie importante ${ }^{177}$, et peut-être la totalité, des 12 volumes des Travaux et mémoires de l'IE parus entre 1935 et 1940, en particulier ceux de Paul Hazoumé ${ }^{178}$, Jeanne Cuisinier $^{179}$ et plusieurs ouvrages issus de la mission Griaule ${ }^{180}$.

\section{Les nouveaux territoires des sciences sociales}

Le paradigme de la recherche expérimentale n'a pas pour seul effet de permettre le renouvellement méthodologique de disciplines existantes, mais également l'affirmation de nouvelles spécialités, voire de nouvelles disciplines, qui y trouvent un moyen de se définir un territoire intellectuel. II témoigne ainsi de la recomposition du paysage des sciences sociales qui s'opère au cours des années trente.

\footnotetext{
169 Griaule à Cavalier, 6 octobre 1934, AN F17/17272.

170 Jean Jamin, "Objets trouvés des paradis perdus, à propos de la mission Dakar-Djibouti », Jacques Hainard et Roland Kaehr (ed), Collections passions, Neufchatel, Musée d'ethnographie, 1982, p. 69.

${ }_{171}^{17}$ Marcel Griaule, « Les résultats de la mission Dakar-Djibouti », Aethiopica, n¹, avril 1933.

172 Jean Jamin, art. cité, p. 69.

173 Marcel Griaule, art. cité.

174 Charles Le Cœur, Mission au Tibesti. Carnets de route 1933-1934, édités par Marguerite Le Cœur, Paris, éditions du CNRS, 1969, p. 7.

${ }^{175}$ Charles Le Cœur, Le rite et l'outil. Essai sur le rationalisme social et la pluralité des civilisations, Paris, Alcan, 1939, p. 82.

176 Denis Paulme, Organisation sociale des dogons (Soudan français), thèse de doctorat, faculté de droit de Paris, 1940.

${ }^{177}$ Rivet à Charléty, 7 mai 1934, CAC 20010498/70.

${ }_{178}$ Le pacte de sang au Dahomey, Paris, Travaux et mémoires de l'Institut d'ethnologie, 1937.

179 Danses magiques de Kelantan, Paris, Travaux et mémoires de l'Institut d'ethnologie, 1936.

180 Les flambeurs d'homme, Paris, 1934 (préface de Sébastien Charléty) ; Jeux Dogons, Paris, Travaux et mémoires de l'Institut d'ethnologie, 1938 ; Sylvain Grébault, Catalogue des manuscrits éthiopiens de la collection Griaule, Paris, 1938.
} 
On peut en voir une illustration avec les enquêtes sur les pratiques religieuses, qui vont contribuer à dilater le territoire de la sociologie religieuse jusque-là consacré essentiellement à l'étude des religions primitives, et ce, grâce à l'apport des recherches menées par le juriste Gabriel Le Bras: amorcées depuis 1931, ses travaux sont aidés à partir de 1935 par le CURS jusqu'en 1938, et classées dans la rubrique "sociologie ». Les grands axes de ce programme d'enquêtes de terrain menées diocèse par diocèse sont tracés dans une conférence à l'Institut français de sociologie publiée dans les Annales Sociologiques ${ }^{181}$, ainsi que dans une intervention au deuxième congrès d'histoire ecclésiastique de la France tenu en mai $1937^{182}$. Elles font l'objet d'une série d'articles intitulés « Notes de statistiques et d'histoire religieuse » dans la Revue d'histoire de l'église de France en 1932, 1933, 1936, 1938 et 1940, et donnent lieu à une première synthèse en $1942^{183}$.

On peut faire la même remarque pour les études de folklore, dont relèvent les trois enquêtes de la CRC financées par le CURS, et coordonnées par André Varagnac: cet ancien élève de Mauss a fondé en 1928 la Société du folklore français puis a approfondi sa réflexion entre 1932 et 1936 dans le cadre d'une bourse de recherches de la CNS obtenue par l'entremise de Bouglé ${ }^{184}$, tout en devenant secrétaire de la $\mathrm{CRC}$ en 1934. Ces enquêtes collectives et quantitatives lui permettent d'affirmer que le folklore, qu'il considère comme une "branche de la sociologie descriptive ", devient une "discipline vraiment scientifique " faisant appel à la statistique " et maniant "la critique des témoignages ${ }^{185}$ ". Cette volonté de constituer un champ disciplinaire à part entière est officialisée en juin-juillet 1937 avec la tenue à Paris du premier congrès international de folklore, et cautionnée par Bouglé, qui charge Varagnac de rédiger dans le volume collectif Les sciences sociales en France un chapitre sur le folklore, qualifié de « dernière née des sciences sociales » ${ }^{186}$.

Les travaux menés par l'ISRES témoignent du même processus à l'œuvre à l'échelle de la discipline économique. Alors que celle-ci n'a encore aucune existence institutionnelle puisqu'elle n'est qu'une branche du droit, l'affirmation d'une posture épistémologique est une étape importante dans son émancipation intellectuelle. De ce point de vue, l'objectif affiché par l'ISRES dans ses statuts est clair: " faire progresser l'emploi des méthodes scientifiques dans l'étude des problèmes économiques et sociaux ${ }^{187}$ ", et l'ensemble de ses travaux reposent sur la collecte empirique de données et leur traitement statistique, depuis la Chronologie économique internationale publiée chaque année à partir de 1934, jusqu'à l'enquête sur le chômage en passant par les nombreuses analyses parues dans L'Activité économique ${ }^{188}$. L'histoire de l'ISRES est, à bien des égards, celle de la construction d'un territoire intellectuel qui participe à l'émancipation de l'économie par rapport au droit et à la sociologie durkheimienne. L'influence de celle-ci est en effet patente dans les premiers travaux de l'institut, non seulement parce que le cadre intellectuel

\footnotetext{
181 Gabriel Le Bras, "Les transformations religieuses des campagnes françaises depuis la fin du XVIle siècle », Annales Sociologiques, Série E, fascicule 2, 1937, pp. 15-70.

${ }_{182}$ Gabriel Le Bras, "Pour l'étude de la paroisse rurale », Revue d'histoire de l'église de France, octobre-décembre 1937, pp. 485-502.

${ }^{183} \mathrm{Id}$, Introduction à l'histoire de la pratique religieuse en France, Paris, Presses universitaires de France, 1942.

${ }_{184}^{184}$ André Varagnac, Définition du folklore, Paris, 1938.

185 Georges-Henri Rivière et André Varagnac, «Le premier congrès international de folklore ", Annales d'histoire économiques et sociales, mars 1937, p. 195.

${ }^{186}$ CEPE, Les Sciences sociales en France, Paris, Hartmann, 1937, p. 118.

187 Statuts de l'ISRES, RF 1.1/500S/21/220.

${ }^{188}$ Ludovic tournès, « L'institut scientifique... » et « Le durkheimisme... », art. cités.
} 
de l'enquête sur le chômage, initiée par Bouglé, est principalement conçu par les représentants de la sociologie économique (Maurice Halbwachs et François Simiand), mais aussi parce qu'à partir de 1934, Robert Marjolin et Philippe Schwob passent du CDS à I'ISRES ${ }^{189}$, où ils deviennent attachés de recherches à plein temps jusqu'en 1939 et dont il constitueront les chevilles ouvrières. L'évolution intellectuelle de Marjolin est à cet égard significative : jusqu'en 1936, il reconnaît la pensée de Durkheim comme un cadre intellectuel de référence ${ }^{190}$ et rédige de nombreux compte rendus de sociologie générale pour les Annales sociologiques. En 1934, il entreprend une thèse d'économie dans le sillage de François Simiand, mais se démarque progressivement de lui ${ }^{191}$, un éloignement qui devient rapidement critique radicale, la dernière partie de sa thèse soutenue en 1941 étant consacrée à la mise en pièce des thèses de Simiand sur la rationalité du mouvement des prix ${ }^{192}$. Cette évolution individuelle reflète celle des travaux de l'ISRES dans leur ensemble, en particulier l'enquête sur le chômage, dont le cadre de référence, initialement durkheimien, est méthodiquement déconstruit quelques années plus tard par les conclusions de l'enquête qui, en reconnaissant le paradigme de l'homo oeconomicus comme un cadre de référence, témoignent clairement d'une rupture intellectuelle avec la sociologie économique durkheimienne.

Les relations internationales illustrent enfin le cas d'une discipline en gestation, dans laquelle le paradigme de la recherche expérimentale joue un rôle unificateur. Tout comme I'ISRES, l'objectif du CEPE est de développer la recherche dans le domaine des relations internationales en mettant en œuvre une « méthode scientifique et [un] appel à l'expérience concrète ${ }^{193}$ ». Dans cette discipline en construction, sans attache institutionnelle solide, et regroupant des chercheurs venus de tous horizons (économistes, géographes, ethnologues, historiens, juristes), la collecte de matériaux de première main et leur traitement quantitatif sous forme statistique sont à la fois une garantie de scientificité, un ferment d'unité intellectuelle et la condition indispensable pour que s'établisse un dialogue entre universitaires et hommes de terrain (diplomates, administrateurs, hommes d'affaires...). Travail de terrain et statistiques constituent donc le sous-bassement de tous les travaux du CEPE, que ce soit la Chronologie politique internationale publiée à partir de 1934, mais aussi les travaux consacrés aux questions économiques, notamment coloniales: ainsi des ouvrages de Charles Robequain et de Pierre Gourou sur l'évolution économique de I'Indochine ${ }^{194}$, celui d'Henri Labouret sur le Cameroun ${ }^{195}$, de Gilbert Maroger sur les revendications coloniales ${ }^{196}$, ou de Roger Lévy sur les échanges commerciaux franco-japonais $^{197}$; mais c'est le cas aussi des ouvrages portant sur l'Allemagne

\footnotetext{
189 Bouglé à Charléty, 9 juillet 1934, AN 61AJ/97.

190 Annales sociologiques, série A, fascicule 2, 1936, p. 147.

191 «Rationalité ou irrationalité des mouvements économiques de longue durée », Annales sociologiques, série D, fasicule 3, 1938, pp. 1-38.

192 Robert Marjolin, Prix, monnaie et production. Essai sur les mouvements économiques de longue durée, thèse de doctorat en Droit, Paris, 1941.

${ }^{193}$ CEPE, Rapport 1935-1936.

194 Charles Robequain, L'évolution économique de l'Indochine française, Paris, Hartmann, 1939 ; Pierre Gourou, L'utilisation du sol en Indochine française, paris, Hartmann, 1939.

${ }^{195}$ Henri Labouret, Le Cameroun, Paris, Hartmann, 1937.

196 Gilbert Maroger, L'Europe et la question coloniale. Revendications coloniales allemandes. Aspirations coloniales polonaises, Paris, Sirey, 1938.

197 Roger Lévy, Les conséquences du développement économique du Japon pour l'empire français, Paris, Hartmann, 1936.
} 
nazie réalisés par le groupe d'études germaniques ${ }^{198}$, qui englobent à la fois les questions juridiques, économiques, sociales, politiques et culturelles.

En 1939, alors que la subvention quinquénale entre dans sa dernière année, la fondation Rockefeller manifeste son intention de poursuivre la collaboration avec les universitaires français : le nouveau directeur de la SSD, Joseph $\mathrm{H}$. Willits, projette de fusionner les budgets des trois structures ${ }^{199}$, et de favoriser le rapprochement entre le CURS et la section des sciences humaines de la CNRS (qui devient le CNRS en octobre 1939), pour aboutir à un unique organisme « qui coordonnerait l'ensemble de la politique en matière de sciences sociales ${ }^{200} »$. Fin avril 1940, il décide de venir en France pour en parler avec Charléty ${ }^{201}$, mais l'invasion allemande stoppe le projet. L'histoire des relations entre la philanthropie américaine et les sciences sociales française ne se termine pourtant pas là, on le sait, puisqu'elle se poursuit après 1945, à travers la création de la Vle section de École pratique des hautes études, dans un autre contexte et avec d'autres acteurs, mais avec la même volonté d'organisation collective et en prenant appui sur l'acquis de l'entre-deux-guerres. Bien que cette suite soit connue, il faut cependant se garder d'interpréter ce qui se passe avant 1939 à l'aune de l'après 1945. La période de l'entre-deux-guerres possède en effet son unité, et son étude en tant que telle apporte plusieurs éléments, tant à l'histoire des activités internationales de la philanthropie américaine qu'à I'histoire des sciences sociales, mais aussi à l'histoire des politiques scientifiques. Elle témoigne d'abord de la densité des réseaux tissés sur les terrains nationaux par la fondation Rockefeller, une dimension fréquemment négligée par les historiens de la philanthropie majoritairement attentifs à la dimension institutionnelle des financements réalisés par les grandes fondations. D'autre part, il est clair que l'on assiste à l'émergence, au cours des années trente, d'un champ des sciences sociales dont l'unité réside à la fois dans l'affirmation du paradigme de la recherche expérimentale et dans la politique de coordination menée conjointement par les universitaires français et la fondation Rockefeller. On ajoutera que l'analyse des arcanes de la politique philanthropique suggère également la nécessité d'explorer plus avant les relations entre sciences de la nature et sciences sociales, tant en ce qui concerne l'élaboration des pratiques de recherche que la construction des paradigmes ou encore l'analyse des réseaux professionnels. Enfin, et surtout, l'étude des négociations entre la fondation et les universitaires français incite à relire les

\footnotetext{
${ }^{198}$ Robert Pelloux, Le parti national socialiste et ses rapports avec l'Etat, Paris, Hartmann, 1936 ; E. Wernert, L'art dans le Ille Reich. Une tentative d'esthétique dirigée, Paris, Hartmann, 1936 ; André Piatier, Le contrôle des devises dans l'économie du IIle Reich, Paris, Hartmann, 1937 ; Jacques Doublet, Le front du travail allemand, Paris, Hartmann, 1937 ; Henry Laufenburger et Pierre Pfimlin, La nouvelle structure économique du Reich : groupes, cartels et politique des prix, Paris, Hartmann, 1938 ; Hubert Beuve-Méry, Vers la plus grande Allemagne, Paris, Hartmann, 1939. Ce dernier ouvrage n'est pas issu des séances du groupe de travail, mais il s'inscrit dans la même thématique.

199 Rapport du CURS 1938-1939, CAC 20010498/111; Charléty à?, 4 juin 1940, CAC $20010498 / 112$.

${ }^{200}$ Joseph H. Willits, Social Science program and policy. Notes on Europe, juin 1939, 3.1/900/23/175

${ }^{201}$ Kittredge à Charléty, 25 avril 1940, CAC 20010498/112.
} 
phénomènes de circulation internationale d'idées et de pratiques scientifiques en adoptant un point de vue prenant en compte le croisement des dynamiques respectives des acteurs en présence ${ }^{202}$. En effet, l'analyse montre bien que l'on a affaire à une pluralité de négociations et non à un simple face à face francoaméricain ; à une construction progressive et conjointe et non à une diffusion d'un projet préalablement élaboré dans le vase clos de l'organisation américaine; à une rencontre de deux dynamiques fortes et non à la réception puis la réinterprétation d'un projet étranger; au lien organique entre les dimensions financière, organisationnelle et intellectuelle et non à la dichotomie entre l'opération technique gérée par les philanthropes, d'un côté, et la mise en œuvre intellectuelle assurée par les universitaires, de l'autre. Tous ces éléments, que l'analyse du long processus de négociations met en valeur, témoignent du rôle majeur joué par la fondation Rockefeller dans l'organisation des sciences sociales en France au cours de l'entredeux-guerres, et montrent dans le même temps l'impossibilité de réduire ce rôle à une exportation pure et simple, que les Français se seraient laissés imposer ou à laquelle ils auraient résisté. Dans le pôle intellectuel franco-rockefellerien composé du CURS, de I'ISRES et du CEPE, se coalisent une vision globale et collective des sciences sociales, un projet intellectuel appuyé sur une posture épistémologique et des thèmes d'études, une organisation favorisant le travail collectif et l'interdisciplinarité, et enfin un financement à la hauteur de ses ambitions intellectuelles. Mais ici, la Rockefeller n'a rien créé ni exporté ; cependant, par sa vision globalisante des sciences sociales, son action à l'échelle de plusieurs disciplines, sa conception utilitariste de la production du savoir, sa collaboration concomitante à des constructions intellectuelles et institutionnelles dans le monde académique américain et mondial, et enfin par ses disponibilités financières considérables, elle a donné une impulsion décisive à l'entreprise de fédération technique et intellectuelle des sciences sociales dont le projet a également émergé chez les universitaires français après 1918, mais ne s'est réellement affirmé qu'à partir du début des années trente.

\footnotetext{
${ }^{202}$ Voir sur ce point Michael Werner \& Bénédicte Zimmermann (dir.), De la comparaison à l'histoire croisée, Paris, Le seuil, 2004.
} 